\title{
Orthogonal and Biorthogonal FIR Hexagonal Filter Banks with Sixfold Symmetry
}

\author{
Qingtang Jiang
}

\begin{abstract}
Recently hexagonal image processing has attracted attention. The hexagonal lattice has several advantages in comparison with the rectangular lattice, the conventionally used lattice for image sampling and processing. For example, a hexagonal lattice needs fewer sampling points; it has better consistent connectivity; it has higher symmetry; its structure is plausible to human vision systems. The multiresolution analysis method has been used for hexagonal image processing. Since the hexagonal lattice has high degree of symmetry, it is desirable that the hexagonal filter banks designed for multiresolution hexagonal image processing also have high order of symmetry which is pertinent to the symmetry structure of the hexagonal lattice. The orthogonal or prefect reconstruction (PR) hexagonal filter banks which are available in the literature have only 3 -fold symmetry. In this paper we investigate the construction of orthogonal and PR FIR hexagonal filter banks with 6-fold symmetry. We obtain block structures of 7-size refinement (7-channel 2-D) orthogonal and PR FIR hexagonal filter banks with 6-fold rotational symmetry. $\sqrt{7}$-refinement orthogonal and biorthogonal wavelets based on these block structures are constructed. In this paper, we also consider FIR hexagonal filter banks with axial (line) symmetry, and we present a block structure of FIR hexagonal filter banks with pseudo 6-fold axial symmetry.
\end{abstract}

Index Terms-Hexagonal lattice, hexagonal image, filter bank with 6-fold symmetry, orthogonal hexagonal filter bank, biorthogonal hexagonal filter bank, $\sqrt{7}$-refinement wavelet, 7 -size refinement multiresolution decomposition/reconstruction.

\section{EDICS Category: MRP-FBNK}

\section{INTRODUCTION}

Image processing is commonly carried out on the rectangular lattice since images are conventionally sampled on such a lattice. Image processing on the hexagonal lattice has attracted attention recently. See a square and hexagonal lattices in the left and right parts of Fig. 1. The hexagonal lattice has several advantages in comparison with the rectangular lattice. For example, a hexagonal lattice needs fewer sampling points; it has better consistent connectivity; it has higher symmetry; its structure is plausible to human vision systems [1]-[8]. Thus, the hexagonal lattice has been used in many areas such as edge detection [9], [10] and pattern recognition [11]-[15]. It has also been used in geoscience [16]-[19].

When a hexagonal lattice $\mathcal{L}$ is used for image sampling, each node (site) on $\mathcal{L}$ represents a small hexagonal cell, called

Manuscript received March 7, 2008; revised June 25, 2008. This work was supported by UM Research Board 10/05 and UMSL Research Award 10/06. The associate editor coordinating the review of this manuscript and approving it for publication was Dr. Alper Erdogan.

The author is with the Department of Mathematics and Computer Science, University of Missouri-St. Louis, St. Louis, MO 63121, USA, e-mail: jiangq@umsl.edu ,web: www.cs.umsl.edu/ jiang .

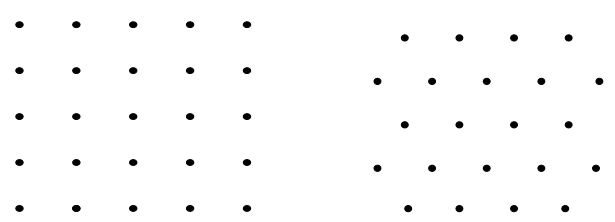

Fig. 1. Square lattice (left) and hexagonal lattice (right)

the elementary cell. A node $b$ and the hexagonal elementary cell (shadowed) it represents are shown in Fig. 2. All the hexagonal elementary cells form a hexagonal tessellation of the plane (see Fig. 2). We call this tessellation to be the hexagonal tessellation associated with $\mathcal{L}$.

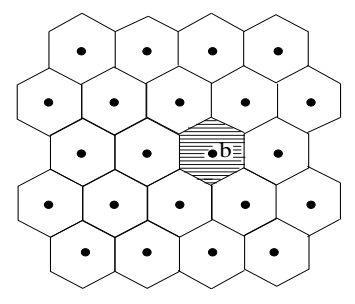

Fig. 2. Hexagonal lattice and associated hexagonal tessellation

It was shown in [20], [21] that a hexagonal lattice allows three interesting refinements (subdivisions): 4-size (4-branch), 3-size (3-branch) and 7-size (7-branch) refinements. As an example, we describe 7 -size refinement ( $\sqrt{7}$-refinement) below. In the left part of Fig. 3, the nodes of the unit regular hexagonal lattice $\mathcal{G}$ are denoted by black dots $\bullet$ and the nodes with circles o form a new lattice, which is called the 7-size (7-branch) sublattice of $\mathcal{G}$ here and it is denoted by $\mathcal{G}_{7} \cdot \mathcal{G}_{7}$ is also a regular hexagonal lattice. From $\mathcal{G}$ to $\mathcal{G}_{7}$, the nodes are reduced by a factor $\frac{1}{7}$. So $\mathcal{G}_{7}$ is a coarse lattice of $\mathcal{G}$, and $\mathcal{G}$ is a refinement of $\mathcal{G}_{7}$. Since $\mathcal{G}_{7}$ is also a regular hexagonal lattice, we can repeat the same procedure to $\mathcal{G}_{7}$, and we then have a highorder (coarse) regular hexagonal lattice with fewer nodes than $\mathcal{G}_{7}$. Repeating this procedure, we have a set of lattices with fewer and fewer nodes. This set of lattices form a "pyramid" or "tree" where a high-order lattice has fewer nodes than its predecessor by a factor of $\frac{1}{7}$. The hexagonal tessellation (with thick hexagon edges) associated with $\mathcal{G}_{7}$ is shown in the right part of Fig. 3, where the hexagonal tessellation (with thin hexagon edges) associated with $\mathcal{G}$ is also provided to give us a picture on how these two tessellations are related to each other. The subdivisions associated with 4-size, 3-size and 7size refinements are called resp. the dyadic (1-to-4 split), $\sqrt{3}$ and $\sqrt{7}$ refinements in the area of Computer Aided Geometry Design [22]-[29], while they are called aperture 4, aperture 3 

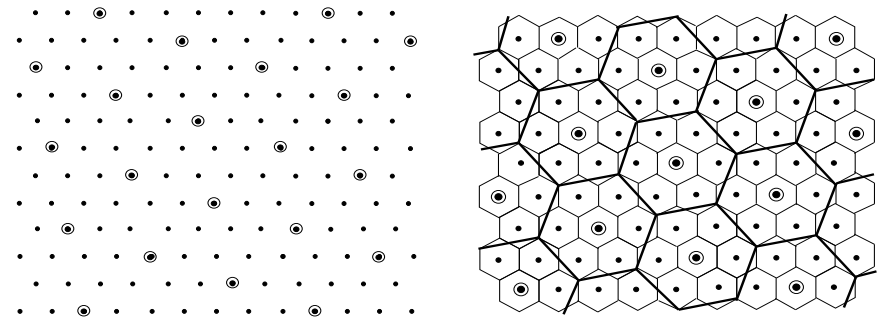

Fig. 3. Hexagonal lattice $\mathcal{G}$ and its 7 -size sublattice $\mathcal{G}_{7}$ (left) and hexagonal tessellations associated with $\mathcal{G}_{7}$ and $\mathcal{G}$ (right)

and aperture 7 (refinements) in discrete global grid systems in [19].

The refinements of the hexagonal lattice allow the multiresolution (multiscale) analysis method to be used to process hexagonally sampled data. The 4-size refinement is the most commonly used refinement for multiresolution image processing, see e.g. [11], [12], [30] for multiresolution hexagonal image processing applications and [30]-[36] for the construction of hexagonal filter banks. The 3 -size refinement is most appealing among these refinements since the 3 -size refinement generates more resolutions and, hence, gives applications more resolutions from which to choose. Compared with 4size and 3-size refinements, the 7-size refinement is not so appealing for multiresolution processing since this refinement results in a reduction in resolution by a factor 7 which is coarse. However, observe from the right picture of Fig. 3 that the hexagonal elementary cells (with thin hexagon edges) associated with $\mathcal{G}$ can be aggregated in groups of seven to form objects which are almost the coarse-resolution hexagonal cells (with thick hexagon edges) associated with $\mathcal{G}_{7}$, while neither $\mathcal{G}_{4}$ nor $\mathcal{G}_{3}$ has such a property. Therefore, the 7size refinement was widely used in planar multiresolution and hexagon-based grid, see [19]. On the other hand, though the 7size refinement multiresolution image processing is considered (see e.g. [37]), the 7-tap orthogonal filter banks in [38] are the only 7-size refinement orthogonal/biorthogonal filter banks available in the literature. These filters were constructed for the purpose of image coding, and their associated wavelets are not continuous, which means that those filters are not suitable for multiresolution image processing since certain smoothness of wavelets is required when the filters are used for multiresolution image processing applications. Therefore, the construction/design of 7-size refinement hexagonal filter banks deserves our investigation.

A regular hexagonal lattice has 6-fold symmetry, while a square lattice has 4-fold symmetry. The feature of higher symmetry for the hexagonal lattice makes image processing more accurate, see [7]. The symmetry of hexagonal filter banks which is closely related to the symmetry structure of the hexagonal lattice is also important for image processing. For example, the symmetry of the hexagonal filter banks in [30] leads to simpler algorithms and efficient computations, see [30]. Therefore, for the hexagonal lattice, it is desirable that the filter banks along it also have 6-fold symmetry. The lowpass filters considered in [30]-[32] do have 6-fold symmetry, but the finite impulse response (FIR) filter banks constructed in these papers are not perfect reconstruction filter banks. The filter banks constructed in [33]-[36] are orthogonal or perfect reconstruction filter banks, but they have only 3-fold symmetry. In our study of 7 -size refinement filter banks, we find that it is possible to construct 7 -size refinement orthogonal FIR hexagonal filter banks with 6-fold symmetry, while one can check directly that it is impossible to construct 6-fold symmetric 4-size or 3-size refinement orthogonal FIR filter banks with reasonable large filter length. The main objective of this paper is to construct 7-size refinement orthogonal and biorthogonal FIR hexagonal filter banks with 6-fold symmetry.

This paper is organized as follows. In Section II, we provide 7-size refinement multiresolution decomposition and reconstruction algorithms and some basic results on the orthogonality/biorthogonality of 7-size refinement filter banks. In Section III, we present a block structure of FIR filter banks with 6fold rotational symmetry. The construction of orthogonal and biorthogonal FIR filter banks of 6-fold rotational symmetry are discussed in Sections IV and V, resp. Finally, in Section VI, we consider FIR filter banks with 6-fold axial symmetry. We provide a condition for a filter bank to have 6 -fold axial symmetry. We find it is hard to obtain a block structure of orthogonal or biorthogonal filter banks with 6-fold axial symmetry. Because of this, we consider in Section VI another type of symmetry, called pseudo 6-fold axial symmetry, and obtain a block structure of FIR filter banks with such a symmetry.

In this paper we use the following notations. For a positive integer $n, I_{n}$ denotes the $n \times n$ identity matrix. For a matrix $M$, we use $M^{*}$ to denote its conjugate transpose $\overline{M^{T}}$, and for a nonsingular matrix $M, M^{-T}$ denotes $\left(M^{-1}\right)^{T}$. We use bold-faced letters such as $\mathbf{x}, \boldsymbol{\omega}$ to denote elements of $\mathbb{R}^{2}$. $\mathbf{x} \cdot \mathbf{y}$ denotes the dot (inner) product $\mathbf{x}^{T} \mathbf{y}$ of $\mathbf{x}=$ $\left[x_{1}, x_{2}\right]^{T}, \mathbf{y}=\left[y_{1}, y_{2}\right]^{T} \in \mathbb{R}^{2}$. For $\mathbf{x}=\left[x_{1}, x_{2}\right]^{T} \in \mathbb{R}^{2}$, denote $d \mathbf{x}=d x_{1} d x_{2}$, and for a function $f$ on $\mathbb{R}^{2}, \hat{f}$ denotes its Fourier transform: $\hat{f}(\boldsymbol{\omega})=\int_{\mathbb{R}^{2}} f(\mathbf{x}) e^{-i \mathbf{x} \cdot \boldsymbol{\omega}} d \mathbf{x}$.

\section{Multiresolution image PROCESSING WiTH 7-SiZE REFINEMENT FILTER BANKS}

In this section, we provide 7-size refinement multiresolution decomposition and reconstruction algorithms and present some basic results on the orthogonality/biorthogonality of 7-size refinement filter banks.

\section{A. 7-size refinement multiresolution algorithms}

Recall that $\mathcal{G}_{7}$ is the coarse lattice of $\mathcal{G}$ after one 7 -size refinement iteration, where $\mathcal{G}$ is the unit regular hexagonal lattice given by

$$
\mathcal{G}=\left\{n_{1} \mathbf{v}_{1}+n_{2} \mathbf{v}_{2}:\left(n_{1}, n_{2}\right) \in \mathbf{Z}^{2}\right\},
$$

where $\mathbf{v}_{1}=[1,0]^{T}, \mathbf{v}_{2}=\left[-\frac{1}{2}, \frac{\sqrt{3}}{2}\right]^{T}$. In general, let $\mathcal{G}_{7^{n}}$ denote the coarse lattice after $n$ steps of 7-size refinement iterations. For an (input) image sampled on $\mathcal{G}$, the nodes of $\mathcal{G}_{7^{n}}$ can be considered as the sampling points of the subsampled image when the multiresolution decomposition algorithm is applied $n$ times to the input image. To provide the multiresolution image decomposition and reconstruction 
algorithms, we need to choose a $2 \times 2$ matrix $N$, called the dilation matrix, such that it maps the hexagonal lattice $\mathcal{G}_{7^{j-1}}$ onto its coarse lattice $\mathcal{G}_{7^{j}}$, namely, $N \mathcal{G}_{7^{j-1}}=\mathcal{G}_{7^{j}}$, where $N \mathcal{G}_{7^{j-1}}=\left\{N \mathbf{g}: \mathbf{g} \in \mathcal{G}_{7^{j-1}}\right\}$. One may choose $N$ to be a matrix that maps $A=\left\{\mathbf{v}_{1}, \mathbf{v}_{2}, \mathbf{v}_{1}+\mathbf{v}_{2},-\mathbf{v}_{1},-\mathbf{v}_{2},-\mathbf{v}_{1}-\mathbf{v}_{2}\right\}$ onto $B=\left\{\mathbf{V}_{1}, \mathbf{V}_{2}, \mathbf{V}_{1}+\mathbf{V}_{2},-\mathbf{V}_{1},-\mathbf{V}_{2},-\mathbf{V}_{1}-\mathbf{V}_{2}\right\}$, where $\mathbf{V}_{1}=2 \mathbf{v}_{1}-\mathbf{v}_{2}, \mathbf{V}_{2}=\mathbf{v}_{1}+3 \mathbf{v}_{2}$. ( $A$ and $B$ are subsets of $\mathcal{G}$ and $\mathcal{G}_{7}$ resp. with their elements forming hexagons.) There are several choices for such a matrix $N$. For example, we may choose $N$ to be one of the matrices:

$$
N_{1}=\left[\begin{array}{cc}
\frac{5}{2} & \frac{\sqrt{3}}{2} \\
-\frac{\sqrt{3}}{2} & \frac{5}{2}
\end{array}\right], \quad N_{2}=\left[\begin{array}{cc}
-\frac{1}{2} & \frac{3 \sqrt{3}}{2} \\
\frac{3 \sqrt{3}}{2} & \frac{1}{2}
\end{array}\right] \text {. }
$$

As sets, both $N_{1} \mathcal{G}_{7^{j-1}}$ and $N_{2} \mathcal{G}_{7^{j-1}}$ are the coarse lattice $\mathcal{G}_{7^{j}}$. But considering the elements of $N_{1} \mathcal{G}_{7^{j-1}}$ and $N_{2} \mathcal{G}_{7^{j-1}}$, one observes that $N_{1} \mathcal{G}_{7^{j-1}}$ keeps the orientation but is rotated clockwise about $19.1^{\circ}$ with respect to the axes of $\mathcal{G}_{7^{j-1}}$ (see the left part of Fig. 4, where $\mathbf{a}_{1}, \cdots, \mathbf{f}_{1}$ are the images of a, $\cdots, \mathbf{f}$ with $N_{1}$ ), while the axes of $N_{2} \mathcal{G}_{7^{j-1}}$ are rotated and reflected from those of $\mathcal{G}_{7^{j-1}}$ (see the right part of Fig. 4, where $\mathbf{a}_{2}, \cdots, \mathbf{f}_{2}$ are the images of $\mathbf{a}, \cdots, \mathbf{f}$ with $\left.N_{2}\right)$. One also observes that the axes of $\left(N_{2}\right)^{2} \mathcal{G}_{7^{j-1}}$ are the same as those of $\mathcal{G}_{7^{j-1}}$ since $\left(N_{2}\right)^{2}$ is $7 I_{2}$. In [39], the subsampling with $N_{1}\left(N_{2}\right.$ resp.) is called the spiraling (toggling resp.) subsampling. For 7-size refinement multiresolution hexagonal image processing, one chooses one of such dilation matrices (and then, keeps using this matrix during the procedure of multiresolution processing). It should be up to one's specific application to use $N_{1}, N_{2}$ or another dilation matrix.

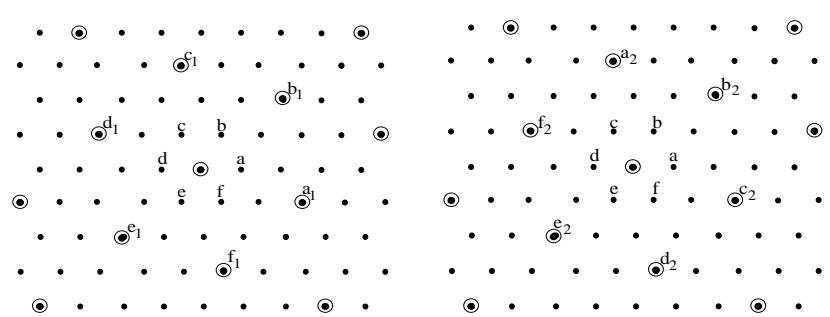

Fig. 4. Hexagonal lattice with spiraling refinement (left) and hexagonal lattice with toggling refinement (right)

For a sequence $\left\{H_{\mathbf{g}}\right\}_{\mathbf{g} \in \mathcal{G}}$ of real numbers associated with $\mathcal{G}$, let $H(\boldsymbol{\omega})$ denote the filter with its impulse response coefficients being $H_{\mathrm{g}}$ (here a factor $1 / 7$ is added for convenience): $H(\boldsymbol{\omega})=(1 / 7) \sum_{\mathbf{g} \in \mathcal{G}} H_{\mathbf{g}} e^{-i \mathbf{g} \cdot \boldsymbol{\omega}}$. Such a filter is called a hexagonal filter. For 7-size refinement multiresolution image processing, a lowpass hexagonal filter $P(\boldsymbol{\omega})$ has 6 associated highpass filters $Q^{(1)}(\boldsymbol{\omega}), \cdots, Q^{(6)}(\boldsymbol{\omega})$.

Let $N$ be a dilation matrix which maps $\mathcal{G}_{7^{j-1}}$ onto $\mathcal{G}_{7^{j}}$. If we use one filter bank $\left\{P, Q^{(1)}, \cdots, Q^{(6)}\right\}$ as the analysis filter bank, and use another filter bank $\left\{\widetilde{P}, \widetilde{Q}^{(1)}, \cdots, \widetilde{Q}^{(6)}\right\}$ as the synthesis filter bank, then the multiresolution (subband) decomposition algorithm with dilation matrix $N$ for an input image $C_{\mathbf{g}, 0}, \mathbf{g} \in \mathcal{G}$ sampled on $\mathcal{G}$ is

$$
\left\{\begin{array}{l}
C_{\mathbf{h}, j+1}=(1 / \sqrt{7}) \sum_{\mathbf{g} \in \mathcal{G}} P_{\mathbf{g}-N \mathbf{h}} C_{\mathbf{g}, j}, \\
D_{\mathbf{h}, j+1}^{(\ell)}=(1 / \sqrt{7}) \sum_{\mathbf{g} \in \mathcal{G}} Q_{\mathbf{g}-N \mathbf{h}}^{(\ell)} C_{\mathbf{g}, j},
\end{array}\right.
$$

with $\ell=1, \cdots, 6, \mathbf{h} \in \mathcal{G}$ for $j=0,1, \cdots, J-1$, where $J$ is a positive integer; and the reconstruction algorithm is

$\hat{C}_{\mathbf{g}, j}=\frac{1}{\sqrt{7}}\left(\sum_{\mathbf{h} \in \mathcal{G}} \widetilde{P}_{\mathbf{g}-N \mathbf{h}} \hat{C}_{\mathbf{h}, j+1}+\sum_{1 \leq \ell \leq 6} \sum_{\mathbf{h} \in \mathcal{G}} \widetilde{Q}_{\mathbf{g}-N \mathbf{h}}^{(\ell)} D_{\mathbf{h}, j+1}^{(\ell)}\right)$

for $j=J-1, J-2, \cdots, 0$, where $\hat{C}_{\mathbf{h}, J}=C_{\mathbf{h}, J}$. If $\hat{C}_{\mathbf{g}, j}=C_{\mathbf{g}, j}, 0 \leq j \leq J-1$ for any input image $C_{\mathbf{g}, 0}, \mathbf{g} \in \mathcal{G}$, then we say that $\left\{P, Q^{(1)}, \cdots, Q^{(6)}\right\}$ and $\left\{\stackrel{P}{P}, \widetilde{Q}^{(1)}, \cdots, \widetilde{Q}^{(6)}\right\}$ are perfect reconstruction filter banks (PR), or that they are biorthogonal filter banks. We say a filter bank $\left\{P, Q^{(1)}, \cdots, Q^{(6)}\right\}$ to be orthogonal if it is biorthogonal to itself.

Assume that $\left\{P, Q^{(1)}, \cdots, Q^{(6)}\right\}$ and $\left\{\widetilde{P}, \widetilde{Q}^{(1)}, \cdots, \widetilde{Q}^{(6)}\right\}$ are two FIR hexagonal filter banks. Let $\Phi$ and $\widetilde{\Phi}$ be the scaling functions (with dilation matrix $N$ ) associated with lowpass filters $P(\boldsymbol{\omega})$ and $\widetilde{P}(\boldsymbol{\omega})$ resp., namely, $\Phi, \widetilde{\Phi}$ satisfy the refinement (two-scale) equations:

$$
\Phi(\mathbf{x})=\sum_{\mathbf{g} \in \mathcal{G}} P_{\mathbf{g}} \Phi(N \mathbf{x}-\mathbf{g}), \widetilde{\Phi}(\mathbf{x})=\sum_{\mathbf{g} \in \mathcal{G}} \widetilde{P}_{\mathbf{g}} \widetilde{\Phi}(N \mathbf{x}-\mathbf{g}) .
$$

Let $\Psi^{(\ell)}, \widetilde{\Psi}^{(\ell)}, 1 \leq \ell \leq 6$, be the functions defined by

$$
\begin{aligned}
& \Psi^{(\ell)}(\mathbf{x})=\sum_{\mathbf{g} \in \mathcal{G}} Q_{\mathbf{g}}^{(\ell)} \Phi(N \mathbf{x}-\mathbf{g}), \\
& \widetilde{\Psi}^{(\ell)}(\mathbf{x})=\sum_{\mathbf{g} \in \mathcal{G}} \widetilde{Q}_{\mathbf{g}}^{(\ell)} \widetilde{\Phi}(N \mathbf{x}-\mathbf{g}) .
\end{aligned}
$$

If $\left\{P, Q^{(1)}, \cdots, Q^{(6)}\right\}$ and $\left\{\widetilde{P}, \widetilde{Q}^{(1)}, \cdots, \widetilde{Q}^{(6)}\right\}$ are biorthogonal to each other, then under certain mild conditions (see the next subsection), $\Phi$ and $\widetilde{\Phi}$ are biorthogonal duals: $\int_{\mathbb{R}^{2}} \Phi(\mathbf{x}) \overline{\widetilde{\Phi}(\mathbf{x}-\mathbf{g})} d \mathbf{x}=\delta_{\mathbf{g}}, \quad \mathbf{g} \in \mathcal{G}$, where $\delta_{\mathbf{g}}$ is the kronecker-delta sequence: $\delta_{\mathbf{g}}=1$ if $\mathbf{g}=(0,0)$, and $\delta_{\mathbf{g}}=0$ if $\mathbf{g} \neq(0,0)$. In this case, $\Psi^{(\ell)}, \Psi^{(\ell)}, 1 \leq \ell \leq 6$, are biorthogonal wavelets, namely, $\left\{\Psi_{j, \mathbf{g}}^{(\ell)}: 1 \leq \ell \leq 6, j \in \mathbf{Z}, \mathbf{g} \in \mathcal{G}\right\}$ and $\left\{\tilde{\Psi}_{j, \mathbf{g}}^{(\ell)}: 1 \leq \ell \leq 6, j \in \mathbf{Z}, \mathbf{g} \in \mathcal{G}\right\}$ are Riesz bases of $L^{2}\left(\mathbb{R}^{2}\right)$ and they are biorthogonal to each other:

$$
\int_{\mathbb{R}^{2}} \Psi_{j, \mathbf{g}}^{(\ell)}(\mathbf{x}) \overline{\widetilde{\Psi}_{j^{\prime}, \mathbf{g}^{\prime}}^{\left(\ell^{\prime}\right)}(\mathbf{x})} d \mathbf{x}=\delta_{j-j^{\prime}} \delta_{\ell-\ell^{\prime}} \delta_{\mathbf{g}-\mathbf{g}^{\prime}},
$$

for $j, j^{\prime} \in \mathbf{Z}, 1 \leq \ell, \ell^{\prime} \leq 6, \mathbf{g}, \mathbf{g}^{\prime} \in \mathcal{G}$, where

$$
\Psi_{j, \mathbf{g}}^{(\ell)}(\mathbf{x})=7^{\frac{j}{2}} \Psi^{(\ell)}\left(N^{j} \mathbf{x}-\mathbf{g}\right), \widetilde{\Psi}_{j, \mathbf{g}}^{(\ell)}(\mathbf{x})=7^{\frac{j}{2}} \widetilde{\Psi}^{(\ell)}\left(N^{j} \mathbf{x}-\mathbf{g}\right) .
$$

For the 7-size refinement, one can obtain biorthogonality condition for the scaling functions $\Phi, \widetilde{\Phi}$ and orthogonality/biorthogonality conditions for hexagonal filter banks. The reader refers to [30], [40] for the biorthogonality conditions for 4-size refinement hexagonal filter banks. Considering that most multiresolution analysis theory and algorithms for image processing are developed along the square lattice $\mathbf{Z}^{2}$ and that most people are more familiar with filter banks on the square lattice, in the next subsection, we give biorthogonality conditions for a pair of $\Phi$ and $\widetilde{\Phi}$ and for a pair of 7-size refinement hexagonal filter banks after we transform them into scaling functions $\phi, \widetilde{\phi}$ and filter banks along $\mathbf{Z}^{2}$.

In the rest of this subsection, we give the definitions of the symmetries of filter banks considered in this paper.

Definition 1: A hexagonal filter bank $\left\{P, Q^{(1)}, \cdots, Q^{(6)}\right\}$ is said to have 6-fold rotational symmetry if its lowpass filter $P(\boldsymbol{\omega})$ is invariant under $\frac{\pi}{3}, \frac{2 \pi}{3}, \pi, \frac{4 \pi}{3}, \frac{5 \pi}{3}$ rotations, and its 
five highpass filters $Q^{(2)}, \cdots, Q^{(6)}$ are $\frac{\pi}{3}, \frac{2 \pi}{3}, \pi, \frac{4 \pi}{3}$ and $\frac{5 \pi}{3}$ (anticlockwise) rotations of the highpass filter $Q^{(1)}$, resp.

Some functions such as 3-direction box-splines $B_{\left[\mathbf{v}_{1}, \cdots, \mathbf{v}_{1}, \mathbf{v}_{2}, \cdots, \mathbf{v}_{2},-\mathbf{v}_{1}-\mathbf{v}_{2}, \cdots,-\mathbf{v}_{1}-\mathbf{v}_{2}\right]}$ along the hexagonal lattice $\mathcal{G}$ have 6-fold axial (line) symmetry, namely, they are symmetric around axes $S_{0}, \cdots, S_{5}$ in $\mathcal{G}$ shown in Fig. 5 (see the symmetry of 3-direction box-splines in [41] at p.114-116, where 6-fold axial symmetry is called the full set of symmetries). It is natural that one considers the construction of scaling functions with such a symmetry. To this regard, we consider hexagonal filter banks with 6-fold axial symmetry.

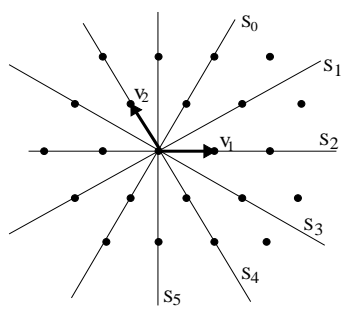

Fig. 5. 6 axes (lines) of symmetry in regular hexagonal lattice

Definition 2: A hexagonal filter bank $\left\{P, Q^{(1)}, \cdots, Q^{(6)}\right\}$ is said to have 6-fold axial symmetry or 6-fold line symmetry if its lowpass filter $P(\boldsymbol{\omega})$ is symmetric around $S_{0}, \cdots, S_{5}$, and its highpass filter $Q^{(1)}$ is symmetric around the axis $S_{0}$ and other five highpass filters $Q^{(2)}, \cdots, Q^{(6)}$ are $\frac{\pi}{3}, \frac{2 \pi}{3}, \pi, \frac{4 \pi}{3}$ and $\frac{5 \pi}{3}$ (anticlockwise) rotations of highpass filter $Q^{(1)}$, resp.

We find that if $\left\{P, Q^{(1)}, \cdots, Q^{(6)}\right\}$ has 6 -fold axial symmetry, then it has 6-fold rotational symmetry, see Section VI. We also find that it is hard to obtain a family of orthogonal or biorthogonal filter banks with 6-fold axial symmetry. Because of this, we consider in Section VI another type of symmetry, called pseudo 6-fold axial symmetry, and provide a family of FIR filter banks with such a symmetry.

\section{B. Transforming the hexagonal lattice to the square lattice $\mathbf{Z}^{2}$}

Let $U$ be the matrix defined by

$$
U=\left[\begin{array}{cc}
1 & \frac{\sqrt{3}}{3} \\
0 & \frac{2 \sqrt{3}}{3}
\end{array}\right]
$$

Then $U$ transforms the regular unit hexagonal lattice $\mathcal{G}$ into the square lattice $\mathbf{Z}^{2}$. For a hexagonal filter $H(\boldsymbol{\omega})=$ $\frac{1}{7} \sum_{\mathbf{g} \in \mathcal{G}} H_{\mathbf{g}} e^{-i \mathbf{g} \cdot \boldsymbol{\omega}}$, after the transformation with the matrix $U$, we have a corresponding filter $h(\boldsymbol{\omega})=\frac{1}{7} \sum_{\mathbf{k} \in \mathbf{Z}^{2}} h_{\mathbf{k}} e^{-i \mathbf{k} \cdot \boldsymbol{\omega}}$ for squarely sampled data with its impulse response $h_{\mathbf{k}}=H_{U^{-1}} \mathbf{k}$. Conversely, for a square filter (filter on the square lattice) $h(\boldsymbol{\omega})=\frac{1}{7} \sum_{\mathbf{k} \in \mathbf{Z}^{2}} h_{\mathbf{k}} e^{-i \mathbf{k} \cdot \boldsymbol{\omega}}$, we have a corresponding hexagonal filter $H(\boldsymbol{\omega})=\frac{1}{7} \sum_{\mathbf{g} \in \mathcal{G}} H_{\mathbf{g}} e^{-i \mathbf{g} \cdot \boldsymbol{\omega}}$ with $H_{\mathbf{g}}=h_{U \mathbf{g}}$.

The matrix $U$ also transforms the scaling functions and wavelets along the hexagonal lattice to those along the square lattice $\mathbf{Z}^{2}$. More precisely, suppose $\left\{P, Q^{(1)}, \cdots, Q^{(6)}\right\}$ and $\left\{\widetilde{P}, \widetilde{Q}^{(1)}, \cdots, \widetilde{Q}^{(6)}\right\}$ are a pair of FIR hexagonal filter banks. Let $\Phi$ and $\widetilde{\Phi}$ be the scaling functions (with dilation matrix $N)$ associated with $P(\boldsymbol{\omega})$ and $\widetilde{P}(\boldsymbol{\omega})$ resp., and $\Psi^{(\ell)}$ and $\widetilde{\Psi}^{(\ell)}, 1 \leq \ell \leq 6$, are the functions defined by (3). Let $\left\{p, q^{(1)}, \cdots, q^{(6)}\right\}$ and $\left\{\widetilde{p}, \widetilde{q}^{(1)}, \cdots, \widetilde{q}^{(6)}\right\}$ be the corresponding square filter banks. Define

$$
\begin{aligned}
& \phi(\mathbf{x})=\Phi\left(U^{-1} \mathbf{x}\right), \psi^{(\ell)}(\mathbf{x})=\Psi^{(\ell)}\left(U^{-1} \mathbf{x}\right), \\
& \widetilde{\phi}(\mathbf{x})=\widetilde{\Phi}\left(U^{-1} \mathbf{x}\right), \widetilde{\psi}^{(\ell)}(\mathbf{x})=\widetilde{\Psi}^{(\ell)}\left(U^{-1} \mathbf{x}\right) \text {. }
\end{aligned}
$$

Then $\phi$ and $\widetilde{\phi}$ are scaling functions satisfying the following refinement equations:

$$
\phi(\mathbf{x})=\sum_{\mathbf{k} \in \mathbf{Z}^{2}} p_{\mathbf{k}} \phi(M \mathbf{x}-\mathbf{k}), \widetilde{\phi}(\mathbf{x})=\sum_{\mathbf{k} \in \mathbf{Z}^{2}} \widetilde{p}_{\mathbf{k}} \widetilde{\phi}(M \mathbf{x}-\mathbf{k}),
$$

and $\psi^{(\ell)}, \widetilde{\psi}^{(\ell)}, 1 \leq \ell \leq 6$ are given by

$$
\begin{aligned}
& \psi^{(\ell)}(\mathbf{x})=\sum_{\mathbf{k} \in \mathbf{Z}^{2}} q_{\mathbf{k}}^{(\ell)} \phi(M \mathbf{x}-\mathbf{k}), \\
& \widetilde{\psi}^{(\ell)}(\mathbf{x})=\sum_{\mathbf{k} \in \mathbf{Z}^{2}} \widetilde{q}_{\mathbf{k}}^{(\ell)} \widetilde{\phi}(M \mathbf{x}-\mathbf{k}),
\end{aligned}
$$

where $p_{\mathbf{k}}, \widetilde{p}_{\mathbf{k}}, q_{\mathbf{k}}^{(\ell)}, \widetilde{q}_{\mathbf{k}}^{(\ell)}$ are exactly the impulse response coefficients of $p(\boldsymbol{\omega}), \widetilde{p}(\boldsymbol{\omega}), q^{(\ell)}(\boldsymbol{\omega}), \widetilde{q}^{(\ell)}(\boldsymbol{\omega})$, resp., and

$$
M=U N U^{-1} \text {. }
$$

Observe that for dilation matrices $N_{1}$ and $N_{2}$ defined by (1), the dilation matrices $M_{1}=U N_{1} U^{-1}$ and $M_{2}=U N_{2} U^{-1}$ for $\phi$ and $\widetilde{\phi}$ are integer matrices:

$$
M_{1}=\left[\begin{array}{cc}
2 & 1 \\
-1 & 3
\end{array}\right], \quad M_{2}=\left[\begin{array}{cc}
1 & 2 \\
3 & -1
\end{array}\right] \text {. }
$$

Equations (6) are the standard (traditional) refinement equations for scaling functions along the commonly used integer lattice $\mathbf{Z}^{2}$ with a (general) integer dilation matrix $M$, and (7) are the standard formulas to define the corresponding wavelets.

For a pair of square filter banks $\left\{p, q^{(1)}, \cdots, q^{(6)}\right\}$ and $\left\{\widetilde{p}, \widetilde{q}^{(1)}, \cdots, \widetilde{q}^{(6)}\right\}$, the traditional multiresolution decomposition and reconstruction algorithms with a dilation matrix $M$ for an input squarely sampled image $c_{\mathbf{k}, 0}$ are (refer to [42])

$$
\left\{\begin{array}{l}
c_{\mathbf{n}, j+1}=(1 / \sqrt{7}) \sum_{\mathbf{k} \in \mathbf{Z}^{2}} p_{\mathbf{k}-M \mathbf{n}} c_{\mathbf{k}, j}, \\
d_{\mathbf{n}, j+1}^{(\ell)}=(1 / \sqrt{7}) \sum_{\mathbf{k} \in \mathbf{Z}^{2}} q_{\mathbf{k}-M \mathbf{n}}^{(\ell)} c_{\mathbf{k}, j},
\end{array}\right.
$$

with $\ell=1, \cdots, 6, \mathbf{n} \in \mathbf{Z}^{2}$ for $j=0,1, \cdots, J-1$, and

$$
\hat{c}_{\mathbf{k}, j}=\frac{1}{\sqrt{7}}\left(\sum_{\mathbf{n} \in \mathbf{Z}^{2}} \widetilde{p}_{\mathbf{k}-M \mathbf{n}} \hat{c}_{\mathbf{n}, j+1}+\sum_{1 \leq \ell \leq 6} \sum_{\mathbf{n} \in \mathbf{Z}^{2}} \widetilde{q}_{\mathbf{k}-M \mathbf{n}}^{(\ell)} d_{\mathbf{n}, j+1}^{(\ell)}\right)
$$

with $\mathbf{k} \in \mathbf{Z}^{2}$ for $j=J-1, J-2, \cdots, 0$, where $\hat{c}_{\mathbf{n}, J}=c_{\mathbf{n}, J}$. We say square filter banks $\left\{p, q^{(1)}, \cdots, q^{(6)}\right\}$ and $\left\{\widetilde{p}, \widetilde{q}^{(1)}, \cdots, \widetilde{q}^{(6)}\right\}$ to be PR filter banks if $\hat{c}_{\mathbf{k}, j}=c_{\mathbf{k}, j}$, $0 \leq j \leq J-1$ for any input squarely sampled image $c_{\mathbf{k}, 0}$.

Clearly, $\left\{P, Q^{(1)}, \cdots, Q^{(6)}\right\}$ and $\left\{\widetilde{P}, \widetilde{Q}^{(1)}, \cdots, \widetilde{Q}^{(6)}\right\}$ are PR filter banks (with $N$ ) if and only if their counterparts $\left\{p, q^{(1)}, \cdots, q^{(6)}\right\}$ and $\left\{\tilde{p}, \widetilde{q}^{(1)}, \cdots, \widetilde{q}^{(6)}\right\}$ are PR filter banks (with $M=U N U^{-1}$ ). From the integer-shift invariant multiresolution analysis theory, we can obtain that $\left\{p, q^{(1)}, \cdots, q^{(6)}\right\}$ and $\left\{\widetilde{p}, \widetilde{q}^{(1)}, \cdots, \widetilde{q}^{(6)}\right\}$ are PR filter banks if and only if

$$
\begin{aligned}
& \sum_{0 \leq k \leq 6} p\left(\boldsymbol{\omega}+2 \pi M^{-T} \boldsymbol{\eta}_{k}\right) \overline{\tilde{p}\left(\boldsymbol{\omega}+2 \pi M^{-T} \boldsymbol{\eta}_{k}\right)}=1 \\
& \sum_{0 \leq k \leq 6} p\left(\boldsymbol{\omega}+2 \pi M^{-T} \boldsymbol{\eta}_{k}\right) \overline{\tilde{q}^{(\ell)}\left(\boldsymbol{\omega}+2 \pi M^{-T} \boldsymbol{\eta}_{k}\right)}=(10) \\
& \sum_{0 \leq k \leq 6} q^{\left(\ell^{\prime}\right)}\left(\boldsymbol{\omega}+2 \pi M^{-T} \boldsymbol{\eta}_{k}\right) \overline{\tilde{q}^{(\ell)}\left(\boldsymbol{\omega}+2 \pi M^{-T} \boldsymbol{\eta}_{k}\right)} \\
& \quad=\delta_{\ell^{\prime}-\ell}
\end{aligned}
$$


for $1 \leq \ell, \ell^{\prime} \leq 6, \boldsymbol{\omega} \in \mathbb{R}^{2}$, where $\boldsymbol{\eta}_{j}, 0 \leq j \leq 6$ are the representatives of the group $\mathbf{Z}^{2} /\left(M^{T} \mathbf{Z}^{2}\right)$ with $\boldsymbol{\eta}_{0}=[0,0]^{T}$. When $M$ is the dilation matrix $M_{1}$ or $M_{2}$ in (8), $\boldsymbol{\eta}_{j}, 1 \leq j \leq 6$ are

$$
\begin{aligned}
& \boldsymbol{\eta}_{1}=[-1,-1]^{T}, \boldsymbol{\eta}_{2}=[0,-1]^{T}, \boldsymbol{\eta}_{3}=[1,0]^{T} \\
& \boldsymbol{\eta}_{4}=[1,1]^{T}, \boldsymbol{\eta}_{5}=[0,1]^{T}, \boldsymbol{\eta}_{6}=[-1,0]^{T}
\end{aligned}
$$

Square filter banks $\left\{p, q^{(1)}, \cdots, q^{(6)}\right\}$ and $\left\{\tilde{p}, \cdots, \tilde{q}^{(6)}\right\}$ are commonly said to be biorthogonal if they satisfy (9)-(11); and a filter bank $\left\{p, q^{(1)}, \cdots, q^{(6)}\right\}$ is commonly referred to be orthogonal if it satisfies (9)-(11) with $\tilde{p}=p, \tilde{q}^{(\ell)}=q^{(\ell)}, 1 \leq$ $\ell \leq 6$.

For an FIR lowpass filter $p(\boldsymbol{\omega})=\frac{1}{7} \sum_{\mathbf{k} \in \mathbf{Z}^{2}} p_{\mathbf{k}} e^{-i \mathbf{k} \cdot \boldsymbol{\omega}}$, let $T_{p}$ denote its transition operator matrix (with dilation matrix $M)$ :

$$
T_{p}=\left[A_{M \mathbf{k}-\mathbf{j}}\right]_{\mathbf{k}, \mathbf{j} \in[-K, K]^{2}},
$$

where $A_{\mathbf{j}}=(1 / 7) \sum_{\mathbf{n} \in \mathbf{Z}^{2}} p_{\mathbf{n}-\mathbf{j}} p_{\mathbf{n}}$ and $K$ is a suitable positive integer depending on the filter length of $p$ and the dilation matrix $M$. We say that $T_{p}$ satisfies Condition $\mathrm{E}$ if 1 is its simple eigenvalue and all other eigenvalues $\lambda$ of $T_{p}$ satisfy $|\lambda|<1$. From the integer-shift invariant multiresolution analysis theory (see e.g. [43], [44], [42]), if $\left\{p, q^{(1)}, \cdots, q^{(6)}\right\}$ and $\left\{\widetilde{p}, \widetilde{q}^{(1)}, \cdots, \widetilde{q}^{(6)}\right\}$ are biorthogonal to each other and the transition operator matrices $T_{p}$ and $T_{\widetilde{p}}$ associated with $p$ and $\widetilde{p}$ satisfy Condition $\mathrm{E}$, then $\phi$ and $\widetilde{\phi}$ are biorthogonal duals: $\int_{\mathbb{R}^{2}} \phi(\mathbf{x}) \widetilde{\phi}(\mathbf{x}-\mathbf{k}) d \mathbf{x}=\delta_{\mathbf{k}}, \mathbf{k} \in \mathbf{Z}^{2}$; and $\psi^{(\ell)}, \psi^{(\ell)}, 1 \leq$ $\ell \leq 6$, defined by (7) are biorthogonal wavelets, namely, $\left\{\psi_{j, \mathbf{k}}^{(\ell)}: 1 \leq \ell \leq 6, j \in \mathbf{Z}, \mathbf{k} \in \mathbf{Z}^{2}\right\}$ and $\left\{\tilde{\psi}_{j, \mathbf{k}}^{(\ell)}: 1 \leq \ell \leq\right.$ $\left.6, j \in \mathbf{Z}, \mathbf{k} \in \mathbf{Z}^{2}\right\}$ are biorthogonal bases of $L^{2}\left(\mathbb{R}^{2}\right)$, where $\psi_{j, \mathbf{k}}^{(\ell)}(\mathbf{x})=7^{\frac{j}{2}} \psi^{(\ell)}\left(M^{j} \mathbf{x}-\mathbf{k}\right), \widetilde{\psi}_{j, \mathbf{k}}^{(\ell)}(\mathbf{x})=7^{\frac{j}{2}} \widetilde{\psi}^{(\ell)}\left(M^{j} \mathbf{x}-\mathbf{k}\right)$.

From the above discussion, we know that to design orthogonal/biorthogonal hexagonal filter banks, we need only to construct square filter banks $\left\{p, q^{(1)}, \cdots, q^{(6)}\right\}$ and $\left\{\widetilde{p}, \widetilde{q}^{(1)}, \cdots, \widetilde{q}^{(6)}\right\}$ such that they satisfy (9)-(11). As a consequence, $\left\{P, Q^{(1)}, \cdots, Q^{(6)}\right\}$ and $\left\{\widetilde{P}, \widetilde{Q}^{(1)}, \cdots, \widetilde{Q}^{(6)}\right\}$ with impulse responses $P_{\mathbf{g}}=p_{U \mathbf{g}}, Q_{\mathbf{g}}^{(\ell)}=q_{U \mathbf{g}}^{(\ell)}, \widetilde{P}_{\mathbf{g}}=\widetilde{p}_{U \mathbf{g}}, \widetilde{Q}_{\mathbf{g}}^{(\ell)}=$ $\widetilde{q}_{U \mathrm{~g}}^{(\ell)}$ are orthogonal/biorthogonal hexagonal filter banks. Furthermore, if the transition operator matrices $T_{p}$ and $T_{\widetilde{p}}$ associated with the square lowpass filters $p$ and $\widetilde{p}$ satisfy Condition $\mathrm{E}$ (and, hence, scaling functions $\phi, \widetilde{\phi}$ associated with $p(\boldsymbol{\omega}), \widetilde{p}(\boldsymbol{\omega})$ form biorthogonal duals and $\psi^{(\ell)}, \widetilde{\psi}^{(\ell)}, 1 \leq \ell \leq 6$ defined by (7) generate biorthogonal wavelets systems), then the hexagonal scaling functions $\Phi, \widetilde{\Phi}$ associated with $P(\boldsymbol{\omega}), \widetilde{P}(\boldsymbol{\omega})$ form biorthogonal duals and $\Psi^{(\ell)}, \widetilde{\Psi}^{(\ell)}, 1 \leq \ell \leq 6$ defined by (3) generate biorthogonal wavelet systems. In addition, if one wants to design hexagonal filters such that the corresponding (hexagonal) scaling functions $\Phi, \widetilde{\Phi}$ and wavelets $\Psi^{(\ell)}, \widetilde{\Psi}^{(\ell)}, 1 \leq \ell \leq 6$ have desirable certain properties such as approximation power, smoothness and nice time-frequency localizations, then one needs only to consider such properties for the scaling functions $\phi, \widetilde{\phi}$ and wavelets $\psi^{(\ell)}, \widetilde{\psi}^{(\ell)}, 1 \leq \ell \leq 6$ associated with square filters since they are related as in (5).

Remark 1: Since $\mathbf{Z}^{2} / M_{1}^{T} \mathbf{Z}^{2}$ and $\mathbf{Z}^{2} / M_{2}^{T} \mathbf{Z}^{2}$ have the same representatives $\boldsymbol{\eta}_{j}, 0 \leq j \leq 6$, if $\left\{p, q^{(1)}, \cdots, q^{(6)}\right\}$ and $\left\{\tilde{p}, \tilde{q}^{(1)}, \cdots, \tilde{q}^{(6)}\right\}$ are biorthogonal with one of $M_{1}, M_{2}$, say $M_{1}$, then they are also biorthogonal to each other with the other dilation matrix, $M_{2}$. Consequently, if a pair of hexagonal filter banks are biorthogonal with one of dilation matrices $N_{1}, N_{2}$, then they are also biorthogonal to each other with the other dilation matrix.

In the following sections, we consider construction of orthogonal/biorthogonal filter banks with 6-fold symmetry. From Remark 1, we need only to consider one of the dilation matrices $M_{1}, M_{2}$. In the rest of this paper, without loss of generality, we choose $M$ to be $M_{1}$.

\section{FILTER BANKS WITH 6-FOLD ROTATIONAL SYMMETRY}

In this section we consider filter banks with 6-fold rotational symmetry. Let $R(\theta)=\left[\begin{array}{cc}\cos \theta & \sin \theta \\ -\sin \theta & \cos \theta\end{array}\right]$ denote the rotation matrix. Denote

$$
\widetilde{R}_{1}=R\left(\frac{\pi}{3}\right), \quad \widetilde{R}_{j}=\left(\widetilde{R}_{1}\right)^{j}, 1 \leq j \leq 5 .
$$

In other words, $\widetilde{R}_{1}, \cdots, \widetilde{R}_{5}$ are the (clockwise) rotation matrices of $\frac{\pi}{3}, \frac{2 \pi}{3}, \pi, \frac{4 \pi}{3}, \frac{5 \pi}{3}$ resp. Then 6 -fold rotational symmetry of a filter bank $\left\{P, Q^{(1)}, \cdots, Q^{(6)}\right\}$ means that

$$
P_{\widetilde{R}_{j} \mathbf{g}}=P_{\mathbf{g}}, Q_{\mathbf{g}}^{(j+1)}=Q_{\widetilde{R}_{j} \mathbf{g}}^{(1)}, 1 \leq j \leq 5, \mathbf{g} \in \mathcal{G} .
$$

For a hexagonal filter bank $\left\{P, Q^{(1)}, \cdots, Q^{(6)}\right\}$, let $\left\{p, q^{(1)}, \cdots, q^{(6)}\right\}$ be its corresponding square filter bank by the transformation with the matrix $U$ in (4). Namely, the impulse responses $p_{\mathbf{k}}, q_{\mathbf{k}}^{(\ell)}$ of $p(\boldsymbol{\omega}), q^{(\ell)}(\boldsymbol{\omega}), 1 \leq \ell \leq 6$ are $P_{U^{-1} \mathbf{k}}, Q_{U^{-1} \mathbf{k}}^{(\ell)}$, resp. Let $R_{1}, R_{2}, \cdots, R_{5}$ denote the matrices $U \widetilde{R}_{1} U^{-1}, U R_{2} U^{-1}, \cdots, U \widetilde{R}_{5} U^{-1}$, resp. More precisely,

$$
\begin{aligned}
& R_{1}=\left[\begin{array}{cc}
0 & 1 \\
-1 & 1
\end{array}\right], R_{2}=\left[\begin{array}{ll}
-1 & 1 \\
-1 & 0
\end{array}\right], \\
& R_{3}=-I_{2}, R_{4}=-R_{1}, R_{5}=-R_{2} .
\end{aligned}
$$

Then one can show that $P, Q^{(1)}, \cdots, Q^{(6)}$ satisfy (15) if and only if $p, q^{(1)}, \cdots, q^{(6)}$ satisfy

$$
p_{R_{j} \mathbf{k}}=p_{\mathbf{k}}, q_{\mathbf{k}}^{(j+1)}=q_{R_{j} \mathbf{k}}^{(1)}, 1 \leq j \leq 5, \mathbf{k} \in \mathbf{Z}^{2} .
$$

To summarize, we have the following proposition.

Proposition 1: Let $\left\{P, Q^{(1)}, \cdots, Q^{(6)}\right\}$ be a hexagonal filter bank and $\left\{p, q^{(1)}, \cdots, q^{(6)}\right\}$ be its corresponding square filter bank. Then $\left\{P, Q^{(1)}, \cdots, Q^{(6)}\right\}$ has 6 -fold rotational symmetry if and only if $\left\{p, q^{(1)}, \cdots, q^{(6)}\right\}$ satisfies (17).

In the following, for convenience, we say a square filter bank $\left\{p, q^{(1)}, \cdots, q^{(6)}\right\}$ has 6 -fold rotational symmetry if it satisfies (17).

Clearly, (17) is equivalent to

$$
p\left(R_{j}^{-T} \boldsymbol{\omega}\right)=p(\boldsymbol{\omega}), q^{(j+1)}(\boldsymbol{\omega})=q^{(1)}\left(R_{j}^{-T} \boldsymbol{\omega}\right), 1 \leq j \leq 5 .
$$

This and the facts that $R_{j}=R_{1}^{j}, 1 \leq j \leq 5$ and $R_{1}^{6}=I_{2}$, imply the following proposition.

Proposition 2: A filter bank $\left\{p, q^{(1)}, \cdots, q^{(6)}\right\}$ has 6 -fold rotational symmetry if and only if it satisfies

$$
\begin{aligned}
& {\left[p, q^{(1)}, \cdots, q^{(6)}\right]^{T}\left(R_{1}^{-T} \boldsymbol{\omega}\right)=} \\
& M_{0}\left[p(\boldsymbol{\omega}), q^{(1)}(\boldsymbol{\omega}), \cdots, q^{(6)}(\boldsymbol{\omega})\right]^{T},
\end{aligned}
$$


where

$$
M_{0}=\left[\begin{array}{lllllll}
1 & 0 & 0 & 0 & 0 & 0 & 0 \\
0 & 0 & 1 & 0 & 0 & 0 & 0 \\
0 & 0 & 0 & 1 & 0 & 0 & 0 \\
0 & 0 & 0 & 0 & 1 & 0 & 0 \\
0 & 0 & 0 & 0 & 0 & 1 & 0 \\
0 & 0 & 0 & 0 & 0 & 0 & 1 \\
0 & 1 & 0 & 0 & 0 & 0 & 0
\end{array}\right]
$$

Next, we construct the filter bank $\left\{p, q^{(1)}, \cdots, q^{(6)}\right\}$ as the product of appropriate block matrices. Assume that we can write $\left[p(\boldsymbol{\omega}), q^{(1)}(\boldsymbol{\omega}), \cdots, q^{(6)}(\boldsymbol{\omega})\right]^{T}$ as $B\left(M^{T} \boldsymbol{\omega}\right)\left[p_{s}(\boldsymbol{\omega}), q_{s}^{(1)}(\boldsymbol{\omega}), \cdots, q_{s}^{(6)}(\boldsymbol{\omega})\right]^{T}$, where $M \quad$ is $M_{1}$ defined in $(8), B(\boldsymbol{\omega})$ is a $7 \times 7$ matrix whose entries are trigonometric polynomials, and $\left\{p_{s}, q_{s}^{(1)}, \cdots, q_{s}^{(6)}\right\}$ is another FIR filter bank with shorter filter lengths. If both $\left\{p, q^{(1)}, \cdots, q^{(6)}\right\}$ and $\left\{p_{s}, q_{s}^{(1)}, \cdots, q_{s}^{(6)}\right\}$ have 6 -fold rotational symmetry, then Proposition 2 implies that $B(\boldsymbol{\omega})$ satisfies

$$
B\left(M^{T} R_{1}^{-T} \boldsymbol{\omega}\right)=M_{0} B\left(M^{T} \boldsymbol{\omega}\right) M_{0}^{-1},
$$

where $M_{0}$ is the matrix defined by (19).

Denote

$I_{0}(\boldsymbol{\omega})=\left[1, e^{i\left(\omega_{1}+\omega_{2}\right)}, e^{i \omega_{2}}, e^{-i \omega_{1}}, e^{-i\left(\omega_{1}+\omega_{2}\right)}, e^{-i \omega_{2}}, e^{i \omega_{1}}\right]^{T}$.

Then, from $R_{1}^{-T} \boldsymbol{\omega}=\left[\omega_{1}+\omega_{2},-\omega_{1}\right]^{T}$, we have $I_{0}\left(R_{1}^{-T} \boldsymbol{\omega}\right)=$ $\left[1, e^{i \omega_{2}}, e^{-i \omega_{1}}, e^{-i\left(\omega_{1}+\omega_{2}\right)}, e^{-i \omega_{2}}, e^{i \omega_{1}}, e^{i\left(\omega_{1}+\omega_{2}\right)}\right]^{T}$. Thus, $I_{0}(\boldsymbol{\omega})$ satisfies (18). Therefore, 1-tap filter bank $\left\{1, e^{i\left(\omega_{1}+\omega_{2}\right)}, e^{i \omega_{2}}, e^{-i \omega_{1}}, e^{-i\left(\omega_{1}+\omega_{2}\right)}, e^{-i \omega_{2}}, e^{i \omega_{1}}\right\} \quad$ has $\quad 6-$ fold rotational symmetry, and it could be used as the initial filter bank with 6 -fold rotational symmetry.

Denote

$D(\boldsymbol{\omega})=\operatorname{diag}\left(1, e^{i\left(\omega_{1}+\omega_{2}\right)}, e^{i \omega_{2}}, e^{-i \omega_{1}}, e^{-i\left(\omega_{1}+\omega_{2}\right)}, e^{-i \omega_{2}}, e^{i \omega_{1}}\right)$

Then $D\left(M^{T} \boldsymbol{\omega}\right)=\operatorname{diag}\left(1, e^{i\left(3 \omega_{1}+2 \omega_{2}\right)}, e^{i\left(\omega_{1}+3 \omega_{2}\right)}, e^{-i\left(2 \omega_{1}-\omega_{2}\right)}\right.$, $\left.e^{-i\left(3 \omega_{1}+2 \omega_{2}\right)}, e^{-i\left(\omega_{1}+3 \omega_{2}\right)}, e^{i\left(2 \omega_{1}-\omega_{2}\right)}\right)$. On the other hand, with $M^{T} R_{1}^{-T}=\left[\begin{array}{cc}3 & 2 \\ -2 & 1\end{array}\right]$, one has that $D\left(M^{T} R_{1}^{-T} \boldsymbol{\omega}\right)=$ $\operatorname{diag}\left(1, e^{i\left(\omega_{1}+3 \omega_{2}\right)}, e^{-i\left(2 \omega_{1}-\omega_{2}\right)}, e^{-i\left(3 \omega_{1}+2 \omega_{2}\right)}, e^{-i\left(\omega_{1}+3 \omega_{2}\right)}\right.$, $\left.e^{i\left(2 \omega_{1}-\omega_{2}\right)}, e^{i\left(3 \omega_{1}+2 \omega_{2}\right)}\right)$. Therefore $D(\boldsymbol{\omega})$ satisfies (20) and it could be used to build the block matrices. Next we use $B(\boldsymbol{\omega})=B D(\boldsymbol{\omega})$ as the block matrix, where $B$ is a $7 \times 7$ (real) constant matrix. Based on the above discussion, we know that $B(\boldsymbol{\omega})=B D(\boldsymbol{\omega})$ satisfies (20) if and only if $B$ satisfies $M_{0} B M_{0}^{-1}=B$, which is equivalent to the fact that $B$ has the form:

$$
B=\left[\begin{array}{lllllll}
b_{11} & b_{12} & b_{12} & b_{12} & b_{12} & b_{12} & b_{12} \\
b_{21} & b_{22} & b_{23} & b_{24} & b_{25} & b_{26} & b_{27} \\
b_{21} & b_{27} & b_{22} & b_{23} & b_{24} & b_{25} & b_{26} \\
b_{21} & b_{26} & b_{27} & b_{22} & b_{23} & b_{24} & b_{25} \\
b_{21} & b_{25} & b_{26} & b_{27} & b_{22} & b_{23} & b_{24} \\
b_{21} & b_{24} & b_{25} & b_{26} & b_{27} & b_{22} & b_{23} \\
b_{21} & b_{23} & b_{24} & b_{25} & b_{26} & b_{27} & b_{22}
\end{array}\right] .
$$

Based on the above discussion, we reach the following result on the filter banks with 6 -fold rotational symmetry.
Theorem 1: If $\left\{p, q^{(1)}, \cdots, q^{(6)}\right\}$ is given by

$$
\begin{aligned}
& {\left[p(\boldsymbol{\omega}), q^{(1)}(\boldsymbol{\omega}), \cdots, q^{(6)}(\boldsymbol{\omega})\right]^{T}=} \\
& \frac{1}{\sqrt{7}} B_{n} D\left(M^{T} \boldsymbol{\omega}\right) B_{n-1} D\left(M^{T} \boldsymbol{\omega}\right) \cdots B_{1} D\left(M^{T} \boldsymbol{\omega}\right) B_{0} I_{0}(\boldsymbol{\omega})
\end{aligned}
$$

for some $n \in \mathbf{Z}_{+}$, where $I_{0}(\boldsymbol{\omega})$ is defined by (21), and $B_{k}, 0 \leq k \leq n$ are constant matrices of the form (23), then $\left\{p, q^{(1)}, \cdots, q^{(6)}\right\}$ is an FIR filter bank with 6 -fold rotational symmetry.

In the next two sections, we show that the block structure in (24) yields orthogonal and biorthogonal FIR filter banks with 6 -fold rotational symmetry.

\section{ORTHOGONAL FILTER BANKS WITH 6-FOLD ROTATIONAL SYMMETRY}

In this section, we study the construction of orthogonal filter banks with 6 -fold rotational symmetry. For an FIR filter bank $\left\{p, q^{(1)}, \cdots, q^{(6)}\right\}$, denote $q^{(0)}(\boldsymbol{\omega})=p(\boldsymbol{\omega})$. Let $U(\boldsymbol{\omega})$ be a $7 \times 7$ matrix defined by $U(\boldsymbol{\omega})=\left[q^{(\ell)}\left(\boldsymbol{\omega}+\boldsymbol{\eta}_{j}\right)\right]_{0 \leq \ell, j \leq 6}$, where $\boldsymbol{\eta}_{0}, \boldsymbol{\eta}_{1}, \cdots, \boldsymbol{\eta}_{6}$ are given in (12). Then $\left\{p, q^{(1)}, \cdots, q^{(6)}\right\}$ is orthogonal if $U(\boldsymbol{\omega})$ is unitary for all $\boldsymbol{\omega} \in \mathbb{R}^{2}$, that is it satisfies

$$
U(\boldsymbol{\omega}) U(\boldsymbol{\omega})^{*}=I_{7}, \quad \boldsymbol{\omega} \in \mathbb{R}^{2} .
$$

Next, we write $q^{(\ell)}(\boldsymbol{\omega}), 0 \leq \ell \leq 6$ as

$$
\begin{aligned}
& q^{(\ell)}(\boldsymbol{\omega})=\frac{1}{\sqrt{7}}\left(q_{0}^{(\ell)}\left(M^{T} \boldsymbol{\omega}\right)+q_{1}^{(\ell)}\left(M^{T} \boldsymbol{\omega}\right) e^{i\left(\omega_{1}+\omega_{2}\right)}\right. \\
& +q_{2}^{(\ell)}\left(M^{T} \boldsymbol{\omega}\right) e^{i \omega_{2}}+q_{3}^{(\ell)}\left(M^{T} \boldsymbol{\omega}\right) e^{-i \omega_{1}}+q_{4}^{(\ell)}\left(M^{T} \boldsymbol{\omega}\right) e^{-i\left(\omega_{1}+\omega_{2}\right)} \\
& \left.+q_{5}^{(\ell)}\left(M^{T} \boldsymbol{\omega}\right) e^{-i \omega_{2}}+q_{6}^{(\ell)}\left(M^{T} \boldsymbol{\omega}\right) e^{i \omega_{1}}\right)
\end{aligned}
$$

where $q_{k}^{(\ell)}(\boldsymbol{\omega})$ are trigonometric polynomials. Let $V(\boldsymbol{\omega})$ denote the polyphase matrix (with dilation matrix $M$ ) of $\left\{p(\boldsymbol{\omega}), q^{(1)}(\boldsymbol{\omega}), \cdots, q^{(6)}(\boldsymbol{\omega})\right\}$ :

$$
V(\boldsymbol{\omega})=\left[q_{k}^{(\ell)}(\boldsymbol{\omega})\right]_{0 \leq \ell, k \leq 6} .
$$

Clearly,

$$
\left[p(\boldsymbol{\omega}), q^{(1)}(\boldsymbol{\omega}), \cdots, q^{(6)}(\boldsymbol{\omega})\right]^{T}=\frac{1}{\sqrt{7}} V\left(M^{T} \boldsymbol{\omega}\right) I_{0}(\boldsymbol{\omega}),
$$

where $I_{0}(\boldsymbol{\omega})$ is defined by (21). One can verify that the $7 \times 7$ matrix $\frac{1}{\sqrt{7}}\left[I_{0}\left(\boldsymbol{\omega}+2 \pi M^{-T} \boldsymbol{\eta}_{0}\right), I_{0}(\boldsymbol{\omega}+\right.$ $\left.\left.2 \pi M^{-T} \boldsymbol{\eta}_{1}\right), \cdots, I_{0}\left(\boldsymbol{\omega}+2 \pi M^{-T} \boldsymbol{\eta}_{6}\right)\right]$ is unitary for all $\boldsymbol{\omega} \in \mathbb{R}^{2}$, which implies that (25) holds if and only if $V(\boldsymbol{\omega})$ is unitary for all $\boldsymbol{\omega} \in \mathbb{R}^{2}$, namely, $V(\boldsymbol{\omega})$ satisfies

$$
V(\boldsymbol{\omega}) V(\boldsymbol{\omega})^{*}=I_{7}, \quad \boldsymbol{\omega} \in \mathbb{R}^{2} .
$$

Therefore, to construct an orthogonal filter bank $\left\{p, q^{(1)}, \cdots, q^{(6)}\right\}$, we need only to construct a trigonometric polynomial matrix $V(\boldsymbol{\omega})$ which satisfies (27).

If $\left\{p, q^{(1)}, \cdots, q^{(6)}\right\}$ is given by (24), then its polyphase matrix $V(\boldsymbol{\omega})$ is

$$
V(\boldsymbol{\omega})=B_{n} D(\boldsymbol{\omega}) B_{n-1} D(\boldsymbol{\omega}) \cdots B_{1} D(\boldsymbol{\omega}) B_{0} .
$$

Since $D(\boldsymbol{\omega})=\operatorname{diag}\left(1, e^{i\left(\omega_{1}+\omega_{2}\right)}, e^{i \omega_{2}}, e^{-i \omega_{1}}, e^{-i\left(\omega_{1}+\omega_{2}\right)}, e^{-i \omega_{2}}\right.$, $\left.e^{i \omega_{1}}\right)$ is unitary, we know that if constant matrices 
$B_{k}, 0 \leq k \leq n$, are orthogonal, then $V(\boldsymbol{\omega})$ satisfies (27).

Next, we consider the orthogonality of a matrix $B$ of the from (23). To this regard, let $W_{1}$ denote the unitary matrix: $W_{1}=(\sqrt{6} / 6)\left[w^{k j}\right]_{0<k, j \leq 5}$, where $w=e^{\frac{2 \pi i}{6}}=\frac{1}{2}+\frac{\sqrt{3}}{2} i$. With $W=\operatorname{diag}\left(1, W_{1}\right)$, one can get that

$$
W B W^{*}=\left[\begin{array}{ccccc}
b_{11} & \sqrt{6} b_{12} & 0 & \cdots & 0 \\
\sqrt{6} b_{21} & \widetilde{b}_{22} & 0 & \cdots & 0 \\
0 & 0 & \widetilde{b}_{33} & \cdots & 0 \\
\vdots & \vdots & \vdots & \ddots & \vdots \\
0 & 0 & 0 & \cdots & \widetilde{b}_{77}
\end{array}\right] \text {, }
$$

where

$$
\begin{gathered}
\widetilde{b}_{22}=b_{22}+b_{23}+b_{24}+b_{25}+b_{26}+b_{27}, \\
\widetilde{b}_{33}=b_{22}-b_{25}+\frac{1}{2}\left(b_{23}-b_{24}-b_{26}+b_{27}\right) \\
\quad-\frac{\sqrt{3}}{2} i\left(b_{23}+b_{24}-b_{26}-b_{27}\right), \\
\widetilde{b}_{44}=b_{22}+b_{25}-\frac{1}{2}\left(b_{23}+b_{24}+b_{26}+b_{27}\right) \\
\quad-\frac{\sqrt{3}}{2} i\left(b_{23}-b_{24}+b_{26}-b_{27}\right), \\
\widetilde{b}_{55}=b_{22}-b_{23}-b_{25}+b_{24}+b_{26}-b_{27}, \\
\widetilde{b}_{66}=\widetilde{b}_{44}, \widetilde{b}_{77}=\widetilde{b}_{33} .
\end{gathered}
$$

Thus $B$ is orthogonal if and only if $\left[\begin{array}{cc}b_{11} & \sqrt{6} b_{12} \\ \sqrt{6} b_{21} & \widetilde{b}_{22}\end{array}\right]$ is orthogonal and $\left|\widetilde{b}_{j j}\right|=1,3 \leq j \leq 7$, which implies that $b_{11}, b_{12}, \widetilde{b}_{22}$ and $\widetilde{b}_{j j}, j=3,4,5$ can be written as

$$
\begin{aligned}
& b_{11}=s_{0} \cos \theta, \sqrt{6} b_{12}=\sin \theta, \sqrt{6} b_{21}=s_{0} \sin \theta, \\
& \widetilde{b}_{22}=-\cos \theta, \widetilde{b}_{33}=\cos \gamma-i \sin \gamma, \\
& \widetilde{b}_{44}=\cos \zeta-i \sin \zeta, \widetilde{b}_{55}=s_{1},
\end{aligned}
$$

where $s_{0}= \pm 1, s_{1}= \pm 1, \theta, \gamma, \zeta \in \mathbb{R}$. From these equations, we have

$$
\begin{aligned}
& b_{11}=s_{0} \cos \theta, b_{12}=\frac{\sqrt{6}}{6} \sin \theta, b_{21}=s_{0} \frac{\sqrt{6}}{6} \sin \theta, \\
& b_{22}=\frac{1}{6}\left(s_{1}-\cos \theta+2 \cos \gamma+2 \cos \zeta\right), \\
& b_{23}=\frac{1}{6}\left(-s_{1}-\cos \theta+\cos \gamma+\sqrt{3} \sin \gamma-\cos \zeta+\sqrt{3} \sin \zeta\right), \\
& b_{24}=\frac{1}{6}\left(s_{1}-\cos \theta-\cos \gamma+\sqrt{3} \sin \gamma-\cos \zeta-\sqrt{3} \sin \zeta\right), \\
& b_{25}=\frac{1}{6}\left(-s_{1}-\cos \theta-2 \cos \gamma+2 \cos \zeta\right) \\
& b_{26}=\frac{1}{6}\left(s_{1}-\cos \theta-\cos \gamma-\sqrt{3} \sin \gamma-\cos \zeta+\sqrt{3} \sin \zeta\right), \\
& b_{27}=\frac{1}{6}\left(-s_{1}-\cos \theta+\cos \gamma-\sqrt{3} \sin \gamma-\cos \zeta-\sqrt{3} \sin \zeta\right) .
\end{aligned}
$$

Thus an orthogonal matrix $B$ of the form (23) has three parameters. We have therefore the following theorem.

Theorem 2: Suppose $\left\{p, q^{(1)}, \cdots, q^{(6)}\right\}$ is given by (24). If each $B_{k}, 0 \leq k \leq n$ is of the form (23) and its entries $b_{i j}$ are given by (28) for some $\theta_{k}, \gamma_{k}, \zeta_{k}$, then $\left\{p, q^{(1)}, \cdots, q^{(6)}\right\}$ is an orthogonal FIR filter bank with 6 -fold rotational symmetry.

Transforming $\left\{p, q^{(1)}, \cdots, q^{(6)}\right\}$ given in Theorem 2 with the matrix $U$ to the filter banks along the hexagonal lattice, we have a family of orthogonal FIR hexagonal filter banks with 6 -fold rotational symmetry and a block structure. With such a family of orthogonal filter banks, by selecting the free parameters, one can design the filters with desirable properties for one's specific applications. For example, one may consider to design filters with optimum time-frequency localization (refer to [45]-[47] for the design of 1-D matrixvalued filters with optimum time-frequency localization). Here we consider the filters based on the Sobolev smoothness of the associated scaling functions $\phi$. For $s \geq 0$, let $W^{s}$ denote the Sobolev space consisting of functions $f(\mathbf{x})$ on $\mathbb{R}^{2}$ with $\int_{\mathbb{R}^{2}}\left(1+|\boldsymbol{\omega}|^{2}\right)^{s}|\hat{f}(\boldsymbol{\omega})|^{2} d \boldsymbol{\omega}<\infty$.

The Sobolev smoothness of a scaling function $\phi$ can be given by the eigenvalues of the transition operator matrix $T_{p}$ defined in (13), where $p(\boldsymbol{\omega})$ is the lowpass filter associated with $\phi$. More precisely, assume that an FIR lowpass filter $p(\omega)$ has sum rule order $m$ (with dilation matrix $M$ ):

$$
p(0,0)=1, D_{1}^{\alpha_{1}} D_{2}^{\alpha_{2}} p\left(2 \pi M^{-T} \boldsymbol{\eta}_{j}\right)=0,1 \leq j \leq 6,
$$

for all $\left(\alpha_{1}, \alpha_{2}\right) \in \mathbf{Z}_{+}^{2}$ with $\alpha_{1}+\alpha_{2}<m$, where $\boldsymbol{\eta}_{j}, 1 \leq j \leq 6$ are defined by (12), $D_{1}$ and $D_{2}$ denote the partial derivatives with the first and second variables of $p(\boldsymbol{\omega})$ resp. Under some condition, sum rule order is equivalent to the approximation order of $\phi$, see [48]. Let $\sigma_{1}, \sigma_{2}$ be the eigenvalues of the dilation matrix $M$. (When $M$ is $M_{1}$ in (8), $\sigma_{1}=\frac{5}{2}+\frac{\sqrt{3}}{2} i, \sigma_{2}=$ $\frac{5}{2}-\frac{\sqrt{3}}{2} i$.) Denote

$$
\bar{S}_{m}:=\left\{\sigma_{1}^{-\alpha_{1}} \sigma_{2}^{-\alpha_{2}}: \quad \alpha_{1}, \alpha_{2} \in \mathbf{Z}_{+}, \alpha_{1}+\alpha_{2}<m\right\},
$$

and $S_{m}:=\operatorname{spec}\left(T_{p}\right) \backslash \bar{S}_{m}$. Then $\phi$ is in Sobolev space $W^{\log _{7} \rho_{0}^{-1}}$, where

$$
\rho_{0}=\max \left\{|\lambda|: \lambda \in S_{m}\right\} .
$$

See [49], [50] for the details and refer to [24] for algorithms and Matlab routines to find the Sobolev smoothness order.

Remark 2: Observe that sum rule order of a lowpass filter $p(\boldsymbol{\omega})$ with a dilation matrix $M$ is characterized by the values of $p(0,0)$ and $D_{1}^{\alpha_{1}} D_{2}^{\alpha_{2}} p(\boldsymbol{\omega})$ at $2 \pi M^{-T} \boldsymbol{\eta}_{j}, 1 \leq j \leq 6$. Observe that $\left\{M_{1}^{-T} \boldsymbol{\eta}_{j}: 1 \leq j \leq 6\right\}=\left\{M_{2}^{-T} \boldsymbol{\eta}_{j}: 1 \leq j \leq 6\right\}$. Thus if $p(\boldsymbol{\omega})$ has sum rule order $m$ with dilation matrix $M_{1}$, then it also has sum rule order $m$ with dilation matrix $M_{2}$.

Example 1: Let $\left\{p, q^{(1)}, \cdots, q^{(6)}\right\}$ be the orthogonal filter bank with 6 -fold rotational symmetry given by (24) with $n=$ 0: $B_{0} I_{0}(\omega)$. This is a 7-tap filter bank. The lowpass filter $p(\boldsymbol{\omega})$ depends on one free parameter $\theta_{0}$. We can choose this parameter such that the resulting $p(\boldsymbol{\omega})$ has sum rule order 1 . The nonzero coefficients $p_{\mathbf{k}}$ of the resulting $p(\boldsymbol{\omega})$ are

$$
p_{00}=p_{-1-1}=p_{0-1}=p_{10}=p_{11}=p_{01}=p_{-10}=1 .
$$

We find that the leading eigenvalues of $T_{p}$ (with $M=M_{1}$ ) are $1, \frac{3}{7}, \sigma_{1}, \sigma_{2}$. Thus, $\rho_{0}=\frac{3}{7}$. With $-\log _{7}\left(\frac{3}{7}\right)=0.4354$, we know that the corresponding scaling function $\phi$ is in $W^{0.4354}$. We can also choose other parameters $\gamma_{0}, \eta_{0}$ for highpass filters such that the nonzero coefficients $q_{\mathbf{k}}^{(1)}$ of $q^{(1)}(\boldsymbol{\omega})$ are

$$
\begin{aligned}
& q_{00}^{(1)}=1, q_{-1-1}^{(1)}=-\frac{1+5 \sqrt{7}}{6}, \\
& q_{0-1}^{(1)}=q_{10}^{(1)}=q_{11}^{(1)}=q_{01}^{(1)}=q_{-10}^{(1)}=\frac{\sqrt{7}-1}{6} .
\end{aligned}
$$

The resulting scaling function $\phi$ is a charactersitic function of a region with fractal boundary. $\phi$ can be approximated by $\phi_{n}$ defined by

$$
\phi_{n}(\mathbf{x})=\sum_{\mathbf{k} \in \mathbf{Z}^{2}} p_{\mathbf{k}} \phi_{n-1}(M \mathbf{x}-\mathbf{k}), n=1,2, \cdots,
$$


where $\phi_{0}$ is a suitable compactly supported function with $\sum_{\mathbf{k} \in \mathbf{Z}^{2}} \phi_{0}(\mathbf{x}-\mathbf{k})=1$. We show the support of $\phi_{3}$ on the left of Fig. 6 with $\phi_{0}(\mathbf{x})=\chi_{\left[-\frac{1}{2}, \frac{1}{2}\right)^{2}}(\mathbf{x})$.

From Remarks 1 and 2, this filter bank is orthogonal with dilation matrix $M_{2}$ and the resulting $p(\boldsymbol{\omega})$ has sum rule order 1 with $M_{2}$. We check that the associated scaling function, denoted by $\varphi$, is also in $W^{0.4354} . \varphi$ is a characteristic function of a region with fractal boundary. The support of $\varphi_{3}(\mathbf{x})$ is shown on the right of Fig.6, where $\varphi_{n}(\mathbf{x})=\sum_{\mathbf{k} \in \mathbf{Z}^{2}} p_{\mathbf{k}} \varphi_{n-1}\left(M_{2} \mathbf{x}-\right.$ $\mathbf{k}), n=1,2,3$, and $\varphi_{0}(\mathbf{x})=\chi_{\left[-\frac{1}{2}, \frac{1}{2}\right)^{2}}(\mathbf{x})$. See [51], [52] for the regions with fractal boundary called dragon and twin dragon sets generated by the quincunx matrices.
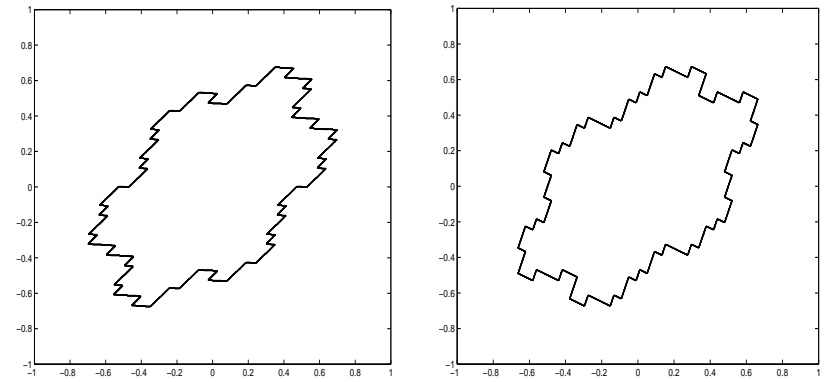

Fig. 6. Approximated support of $\phi$ with dilation $M_{1}$ (left) and that of $\varphi$ with dilation $M_{2}$ (right)
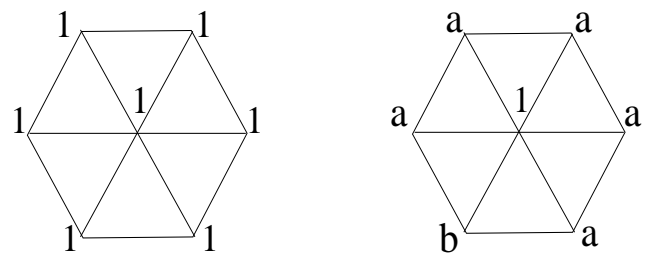

Fig. 7. Impulse responses $P_{\mathbf{g}}$ (left) and $Q_{\mathrm{g}}^{(1)}$ with $a=\frac{\sqrt{7}-1}{6}, b=$ $-\frac{1+5 \sqrt{7}}{6}$ (right), while $Q_{\mathrm{g}}^{(j+1)}$ are $\frac{\pi j}{3}$ rotations of $Q_{\mathrm{g}}^{(1)}, 1 \leq j \leq 5$

Let $\left\{P_{0}, Q_{0}^{(1)}, \cdots, Q_{0}^{(6)}\right\}$ be the corresponding orthogonal hexagonal filter bank. The nonzero impulse response coefficients $P_{\mathbf{g}}, Q_{\mathbf{g}}^{(1)}$ of $P_{0}(\boldsymbol{\omega}), Q_{0}^{(1)}(\boldsymbol{\omega})$ are displayed in Fig. 7, while $Q_{\mathrm{g}}^{(j+1)}$ are $\frac{\pi j}{3}$ rotations of $Q_{\mathrm{g}}^{(1)}, 1 \leq j \leq 5$. Notice that the lowpass filter $P_{0}(\boldsymbol{\omega})$ has 6 -fold axial symmetry, namely, it is symmetric around axes $S_{0}, \cdots, S_{5}$ shown in Fig. 5, and that $Q_{0}^{(1)}(\boldsymbol{\omega})$ is symmetric around axis $S_{0}$. Therefore, this orthogonal filter bank has 6-fold axial symmetry. In [38], three 7-tap orthogonal hexagonal filter banks were constructed for image coding with all filter banks having the same lowpass filter which is exactly $P_{0}(\boldsymbol{\omega})$. The highpass filters in [38] are different from $Q_{0}^{(\ell)}(\boldsymbol{\omega}), 1 \leq \ell \leq 6$. Three of their highpass filters are symmetric around the axis $S_{1}$ and the other three are anti-symmetric around $S_{1}$.

Example 2: Let $\left\{p, q^{(1)}, \cdots, q^{(6)}\right\}$ be the orthogonal filter bank with 6 -fold rotational symmetry given by (24) for $n=1$ : $B_{1} D\left(M^{T} \boldsymbol{\omega}\right) B_{0} I_{0}(\boldsymbol{\omega})$ with $s_{0}, s_{1}$ in (28) being 1 . The lowpass filter $p(\boldsymbol{\omega})$ depends on four parameters $\theta_{0}, \gamma_{0}, \eta_{0}, \theta_{1}$. By solving the equations for sum rule order 2 , we get

$$
\begin{aligned}
& \theta_{0}=\tan ^{-1}(7 \sqrt{6}-\sqrt{258}), \gamma_{0}=-\tan ^{-1}\left(\frac{\sqrt{3}}{5}\right) \\
& \theta_{1}=-\sin ^{-1}\left(\frac{\sqrt{6}}{7}\right)
\end{aligned}
$$

or

$$
\begin{aligned}
& \theta_{0}=\tan ^{-1}(7 \sqrt{6}+\sqrt{258}), \gamma_{0}=\pi-\tan ^{-1}\left(\frac{\sqrt{3}}{5}\right), \\
& \theta_{1}=\sin ^{-1}\left(\frac{\sqrt{6}}{7}\right) .
\end{aligned}
$$

Thus, we have a lowpass filter $p(\boldsymbol{\omega})$ depending on one free parameter $\eta_{0}$. Then we select this free parameter such that $\rho_{0}$ in (30) as small as possible. With $\eta_{0}=\pi-\tan ^{-1}\left(\frac{20}{21}\right)$ and $\theta_{0}, \gamma_{0}, \theta_{1}$ given in (31), we have the (numerically) most smooth scaling function $\phi$ which is in $W^{0.9202}$. There are two free parameters $\gamma_{1}, \eta_{1}$ left for the highpass filters of this orthogonal filter bank. Here we simply set $\gamma_{1}=\eta_{1}=0$. In Fig. 8 we show the pictures of the resulting $\phi$ and $\psi^{(1)}$.
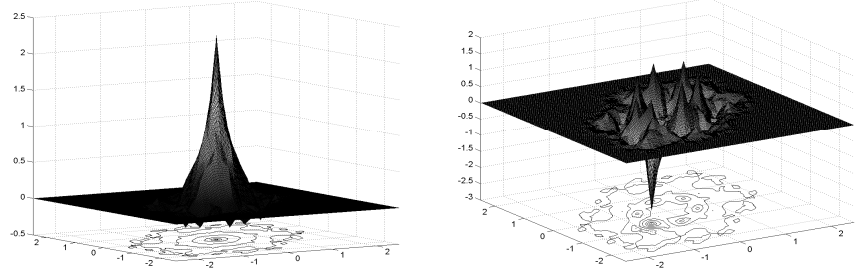

Fig. 8. $\phi$ (left) and $\psi^{(1)}($ right)

Let $\left\{P, Q^{(1)}, \cdots, Q^{(6)}\right\}$ be the corresponding orthogonal hexagonal filter bank. We apply this filter bank to a hexagonally sampled image in Fig. 9. This is a part of the hexagonal image re-sampled from a $512 \times 512$ squarely sampled image Lena by the bilinear interpolation in [6]. The decomposed images with the lowpass filter and highpass filters are shown on the left of Fig. 10 and in Fig. 11 resp. These images are indeed rotated about $19.1^{\circ}$ with respect to the original image. The decomposed image with the lowpass filter applied twice is shown on the right of Fig. 10.

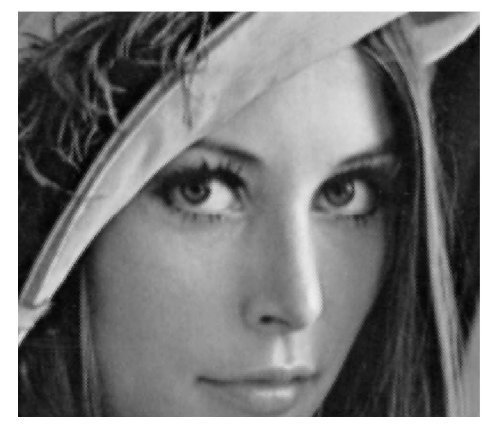

Fig. 9. Original (hexagonal) image

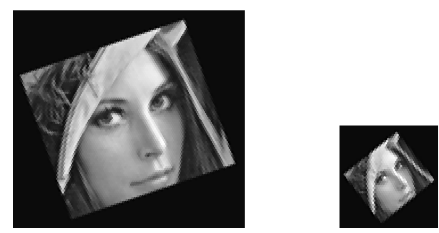

Fig. 10. Decomposed images with lowpass filter $P$ with one (left) and two (right) iterations

We also consider orthogonal filter banks given (24) with a few more blocks $B_{k} D\left(M^{T} \boldsymbol{\omega}\right)$. We find that it is hard to construct orthogonal scaling functions 

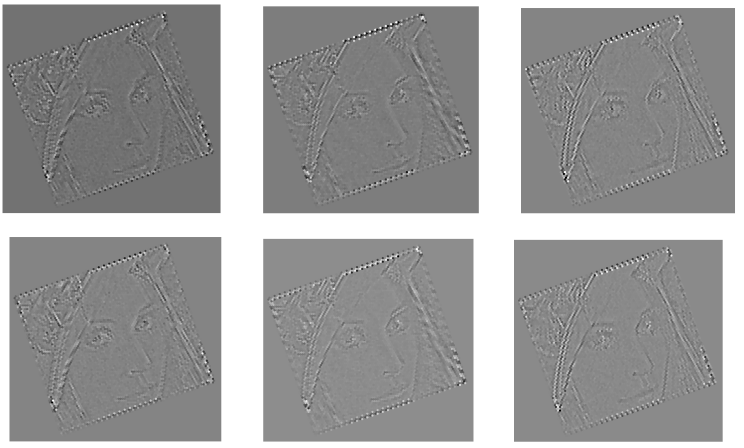

Fig. 11. Decomposed images with highpass filters $Q^{(1)}, Q^{(2)}, Q^{(3)}$ (top row from left) and $Q^{(4)}, Q^{(5)}, Q^{(6)}$ (bottom row from left)

with high smoothness order. For example, it seems that $B_{2} D\left(M^{T} \boldsymbol{\omega}\right) B_{1} D\left(M^{T} \boldsymbol{\omega}\right) B_{0} I_{0}(\boldsymbol{\omega})$ cannot lead to an orthogonal scaling function in $W^{1}$. Thus, to construct orthogonal scaling and wavelets with nice smoothness order, we need to use more blocks $B_{k} D\left(M^{T} \boldsymbol{\omega}\right)$. In the next section, we consider 6 -fold rotational symmetric biorthogonal filter banks, which give us some flexility for the construction of PR filter banks.

\section{BIORTHOGONAL FILTER BANKS WITH 6-FOLD ROTATIONAL SYMMETRY}

In this section we consider biorthogonal filter banks with 6 -fold rotational symmetry. Suppose $\left\{p, q^{(1)}, \cdots, q^{(6)}\right\}$ and $\left\{\tilde{p}, \tilde{q}^{(1)}, \cdots, \widetilde{q}^{(6)}\right\}$ are a pair of filter banks. Let $V(\boldsymbol{\omega})$ and $\widetilde{V}(\boldsymbol{\omega})$ be their polyphase matrices defined as in (26). Then one can obtain as in the above section that $\left\{p, q^{(1)}, \cdots, q^{(6)}\right\}$ and $\left\{\tilde{p}, \tilde{q}^{(1)}, \cdots, \widetilde{q}^{(6)}\right\}$ are biorthogonal to each other if and only if $V(\boldsymbol{\omega})$ and $\widetilde{V}(\boldsymbol{\omega})$ satisfy $V(\boldsymbol{\omega}) \widetilde{V}(\boldsymbol{\omega})^{*}=I_{7}, \boldsymbol{\omega} \in$ $\mathbb{R}^{2}$. If $\left\{p, q^{(1)}, \cdots, q^{(6)}\right\}$ is the FIR filter bank given by (24) for some $7 \times 7$ real matrices $B_{k}$, then $V(\boldsymbol{\omega})=$ $B_{n} D(\boldsymbol{\omega}) B_{n-1} D(\boldsymbol{\omega}) \cdots B_{1} D(\boldsymbol{\omega}) B_{0}$. If the constant matrices $B_{k}, 0 \leq k \leq n$ are nonsingular, then from the fact that $\left(D(\boldsymbol{\omega})^{*}\right)^{-1}=D(\boldsymbol{\omega})$, we know

$$
\left(V(\boldsymbol{\omega})^{*}\right)^{-1}=B_{n}^{-T} D(\boldsymbol{\omega}) B_{n-1}^{-T} D(\boldsymbol{\omega}) \cdots B_{1}^{-T} D(\boldsymbol{\omega}) B_{0}^{-T} .
$$

Clearly if $B_{k}$ has the form of (23), namely, $M_{0} B_{k} M_{0}^{-1}=$ $B_{k}$, then $M_{0} B_{k}^{-T} M_{0}^{-1}=B_{k}^{-T}$ since $M_{0}^{T}=M_{0}^{-1}$. Thus $B_{k}^{-T}$ also has the form of (23). Hence, by Proposition 1, $\left\{\tilde{p}, \tilde{q}^{(1)}, \cdots, \tilde{q}^{(6)}\right\}$ given by

$$
\begin{aligned}
& {\left[\widetilde{p}(\boldsymbol{\omega}), \widetilde{q}^{(1)}(\boldsymbol{\omega}), \cdots, \widetilde{q}^{(6)}(\boldsymbol{\omega})\right]^{T}=} \\
& \quad \frac{1}{\sqrt{7}} B_{n}^{-T} D\left(M^{T} \boldsymbol{\omega}\right) \cdots B_{1}^{-T} D\left(M^{T} \boldsymbol{\omega}\right) B_{0}^{-T} I_{0}(\boldsymbol{\omega})
\end{aligned}
$$

with $I_{0}(\boldsymbol{\omega})$ defined by (21), has 6-fold rotational symmetry. For this filter bank $\left\{\tilde{p}, \tilde{q}^{(1)}, \cdots, \tilde{q}^{(6)}\right\}$, since its polyphase matrix $\widetilde{V}(\boldsymbol{\omega})$ is $\left(V(\boldsymbol{\omega})^{*}\right)^{-1}$, we know it is biorthogonal to $\left\{p, q^{(1)}, \cdots, q^{(6)}\right\}$. To summarize, we have the following theorem.

Theorem 3: Suppose that $\left\{p, q^{(1)}, \cdots, q^{(6)}\right\}$ are the FIR filter bank given by (24) where $B_{k}, 0 \leq k \leq n$ are nonsingular constant matrices of the form (23). Let $\left\{\tilde{p}, \tilde{q}^{(1)}, \cdots, \widetilde{q}^{(6)}\right\}$ be the FIR filter bank given by (33). Then $\left\{\tilde{p}, \tilde{q}^{(1)}, \cdots, \tilde{q}^{(6)}\right\}$ is an FIR filter bank biorthogonal to $\left\{p, q^{(1)}, \cdots, q^{(6)}\right\}$ and it has 6 -fold rotational symmetry.

Theorem 3 provides a family of biorthogonal FIR filter banks with 6 -fold rotational symmetry. Compared with the orthogonal filter banks in Theorem 2, this family of biorthogonal filter banks allows for some flexibility in filter design.

Example 3: Let $\left\{p, q^{(1)}, \cdots, q^{(6)}\right\}$ and $\left\{\tilde{p}, \tilde{q}^{(1)}, \cdots, \widetilde{q}^{(6)}\right\}$ be the biorthogonal filter banks with 6 -fold rotational symmetry given by Theorem 3 with $n=1$. We can choose the free parameters for $B_{0}$ and $B_{1}$ such that the resulting scaling functions $\phi \in W^{1.3801}, \tilde{\phi} \in W^{0.6187}$ with the corresponding lowpass filters $p(\boldsymbol{\omega})$ and $\widetilde{p}(\boldsymbol{\omega})$ having sum rule orders 2 and 1 resp. The selected parameters are provided in Appendix A.

Example 4: Let $\left\{p, q^{(1)}, \cdots, q^{(6)}\right\}$ and $\left\{\tilde{p}, \tilde{q}^{(1)}, \cdots, \widetilde{q}^{(6)}\right\}$ be the biorthogonal filter banks with 6 -fold rotational symmetry given by Theorem 3 with $n=2$. In this case, we can choose the free parameters for $B_{0}, B_{1}$ and $B_{2}$ such that the resulting scaling functions $\phi \in W^{1.9877}, \tilde{\phi} \in W^{0.3562}$. We can also select other parameters such that $\phi \in W^{1.8019}, \tilde{\phi} \in$ $W^{0.7422}$, and we use Bio_2 to denote the resulting pair of biorthogonal filter banks. In either case, the corresponding lowpass filters $p(\boldsymbol{\omega})$ and $\widetilde{p}(\boldsymbol{\omega})$ having sum rule orders 2 and 1 resp. The select other parameters for Bio_2 are provided in Appendix B.

\section{ORTHOGONAL/BIORTHOGONAL FILTER BANKS WITH PSEUDO 6-FOLD AXIAL SYMMETRY}

In Sections III-V, we discuss the construction of filter banks with 6 -fold rotational symmetry. In this section we consider filter banks with 6 -fold axial symmetry.

Denote

$$
\begin{aligned}
& \widetilde{L}_{0}=\left[\begin{array}{cc}
-\frac{1}{2} & \frac{\sqrt{3}}{2} \\
\frac{\sqrt{3}}{2} & \frac{1}{2}
\end{array}\right], \widetilde{L}_{1}=\left[\begin{array}{cc}
\frac{1}{2} & \frac{\sqrt{3}}{2} \\
\frac{\sqrt{3}}{2} & -\frac{1}{2}
\end{array}\right], \\
& \widetilde{L}_{2}=\left[\begin{array}{cc}
1 & 0 \\
0 & -1
\end{array}\right], \widetilde{L}_{3}=-\widetilde{L}_{0}, \widetilde{L}_{4}=-\widetilde{L}_{1}, \widetilde{L}_{5}=-\widetilde{L}_{2} .
\end{aligned}
$$

Let $\widetilde{R}_{j}, 1 \leq j \leq 5$ be the rotational matrices define in (14). Then 6 -fold axial symmetry of $\left\{P, Q^{(1)}, \cdots, Q^{(6)}\right\}$ means that for $0 \leq k \leq 5,1 \leq j \leq 5$,

$$
P_{\widetilde{L}_{k} \mathbf{g}}=P_{\mathbf{g}}, Q_{\widetilde{L}_{0} \mathbf{g}}^{(1)}=Q_{\mathbf{g}}^{(1)}, Q_{\mathbf{g}}^{(j+1)}=Q_{\widetilde{R}_{j} \mathbf{g}}^{(1)}, \mathbf{g} \in \mathcal{G} .
$$

One can verify that $\widetilde{R}_{1}=\widetilde{L}_{1} \widetilde{L}_{0}$. This, together with the fact $\widetilde{R}_{j}=\left(\widetilde{R}_{1}\right)^{j}, 2 \leq j \leq 5$, implies that if $\left\{P, Q^{(1)}, \cdots, Q^{(6)}\right\}$ has 6 -fold axial symmetry, then it has 6 -fold rotational symmetry. On the other hand, from the fact that

$$
\begin{aligned}
& \widetilde{L}_{1}=\widetilde{R}_{1} \widetilde{L}_{0}, \widetilde{L}_{2}=\widetilde{L}_{1} \widetilde{L}_{0} \widetilde{L}_{1}, \widetilde{L}_{3}=\widetilde{L}_{2} \widetilde{L}_{0} \widetilde{L}_{1}, \\
& \widetilde{L}_{4}=\widetilde{L}_{0} \widetilde{L}_{2} \widetilde{L}_{0}, \widetilde{L}_{5}=\widetilde{L}_{0} \widetilde{L}_{1} \widetilde{L}_{0},
\end{aligned}
$$

we know that the condition $P_{\widetilde{L}_{0} \mathrm{~g}}=P_{\mathrm{g}}$ and $P_{\widetilde{R}_{1} \mathrm{~g}}=P_{\mathrm{g}}$ for $P(\boldsymbol{\omega})$ is equivalent to the condition $P_{\widetilde{L}_{k} \mathbf{g}}=P_{\mathbf{g}}, 0 \leq k \leq 5$. Thus we have simpler conditions for the 6 -fold axial symmetry of a filter bank as summarized in the next proposition.

Proposition 3: A hexagonal filter bank $\left\{P, Q^{(1)}, \cdots, Q^{(6)}\right\}$ has 6 -fold axial symmetry if and only if for $1 \leq j \leq 5, \mathrm{~g} \in \mathcal{G}$,

$$
P_{\widetilde{L}_{0} \mathbf{g}}=P_{\widetilde{R}_{1} \mathbf{g}}=P_{\mathbf{g}}, Q_{\widetilde{L}_{0} \mathbf{g}}^{(1)}=Q_{\mathbf{g}}^{(1)}, Q_{\mathbf{g}}^{(j+1)}=Q_{\widetilde{R}_{j} \mathbf{g}}^{(1)} .
$$


For a hexagonal filter bank $\left\{P, Q^{(1)}, \cdots, Q^{(6)}\right\}$, let $\left\{p, q^{(1)}, \cdots, q^{(6)}\right\}$ be the corresponding square filter bank by the transformation with the matrix $U$ in (4). Let $L_{0}$ denote the matrix $U \widetilde{L}_{0} U^{-1}$, namely,

$$
L_{0}=\left[\begin{array}{ll}
0 & 1 \\
1 & 0
\end{array}\right] \text {. }
$$

Then $P, Q^{(1)}, \cdots, Q^{(6)}$ satisfy (35) if and only if $p, q^{(1)}, \cdots, q^{(6)}$ satisfy

$$
p_{L_{0} \mathbf{k}}=p_{R_{1} \mathbf{k}}=p_{\mathbf{k}}, q_{L_{0} \mathbf{k}}^{(1)}=q_{\mathbf{k}}^{(1)}, q_{\mathbf{k}}^{(j+1)}=q_{R_{j} \mathbf{k}}^{(1)},
$$

for $1 \leq j \leq 5, \mathbf{k} \in \mathbf{Z}^{2}$, which is equivalent to

$$
\begin{aligned}
& p\left(L_{0} \boldsymbol{\omega}\right)=p\left(R_{1}^{-T} \boldsymbol{\omega}\right)=p(\boldsymbol{\omega}), \\
& q^{(1)}\left(L_{0} \boldsymbol{\omega}\right)=q^{(1)}(\boldsymbol{\omega}), q^{(j+1)}(\boldsymbol{\omega})=q^{(1)}\left(R_{j}^{-T} \boldsymbol{\omega}\right),
\end{aligned}
$$

for $1 \leq j \leq 5$. From Proposition 2, (36) and the relationship among $L_{0}, R_{j}, 1 \leq j \leq 5$, one can obtain the following proposition (the detail of derivation is omitted here).

Proposition 4: A hexagonal filter bank $\left\{P, Q^{(1)}, \cdots, Q^{(6)}\right\}$ has 6-fold axial symmetry if and only if its corresponding square filter bank $\left\{p, q^{(1)}, \cdots, q^{(6)}\right\}$ satisfies (18) and

$$
\begin{aligned}
& {\left[p, q^{(1)}, \cdots, q^{(6)}\right]^{T}\left(L_{0} \boldsymbol{\omega}\right)=} \\
& N_{0}\left[p(\boldsymbol{\omega}), q^{(1)}(\boldsymbol{\omega}), \cdots, q^{(6)}(\boldsymbol{\omega})\right]^{T}
\end{aligned}
$$

where

$$
N_{0}=\left[\begin{array}{lllllll}
1 & 0 & 0 & 0 & 0 & 0 & 0 \\
0 & 1 & 0 & 0 & 0 & 0 & 0 \\
0 & 0 & 0 & 0 & 0 & 0 & 1 \\
0 & 0 & 0 & 0 & 0 & 1 & 0 \\
0 & 0 & 0 & 0 & 1 & 0 & 0 \\
0 & 0 & 0 & 1 & 0 & 0 & 0 \\
0 & 0 & 1 & 0 & 0 & 0 & 0
\end{array}\right]
$$

Suppose $\left\{p, q^{(1)}, \cdots, q^{(6)}\right\}$ can be written

$$
\begin{aligned}
& {\left[p(\boldsymbol{\omega}), q^{(1)}(\boldsymbol{\omega}), \cdots, q^{(6)}(\boldsymbol{\omega})\right]^{T}=} \\
& C(\boldsymbol{\omega})\left[p_{s}(\boldsymbol{\omega}), q_{s}^{(1)}(\boldsymbol{\omega}), \cdots, q_{s}^{(6)}(\boldsymbol{\omega})\right]^{T},
\end{aligned}
$$

where $C(\boldsymbol{\omega})$ is a $7 \times 7$ matrix with trigonometric polynomial entries, and $\left\{p_{s}, q_{s}^{(1)}, \cdots, q_{s}^{(6)}\right\}$ is another FIR filter bank. If both $\left\{p, q^{(1)}, \cdots, q^{(6)}\right\}$ and $\left\{p_{s}, q_{s}^{(1)}, \cdots, q_{s}^{(6)}\right\}$ satisfy (36), then Proposition 3 implies that $C(\boldsymbol{\omega})$ satisfies

$$
C\left(R_{1}^{-T} \boldsymbol{\omega}\right)=M_{0} C(\boldsymbol{\omega}) M_{0}^{-1}, C\left(L_{0} \boldsymbol{\omega}\right)=N_{0} C(\boldsymbol{\omega}) N_{0}^{-1},
$$

where $M_{0}$ and $N_{0}$ are the matrices defined by (19) and (38) resp.

For $I_{0}(\boldsymbol{\omega})$ defined by (21), we show in Section III that $I_{0}(\boldsymbol{\omega})$ satisfies (18). On the other hand,

$I_{0}\left(L_{0} \boldsymbol{\omega}\right)=\left[1, e^{i\left(\omega_{1}+\omega_{2}\right)}, e^{i \omega_{1}}, e^{-i \omega_{2}}, e^{-i\left(\omega_{1}+\omega_{2}\right)}, e^{-i \omega_{1}}, e^{i \omega_{2}}\right]^{T}$

Thus $I_{0}(\boldsymbol{\omega})$ also satisfies (37). That is, filter bank

$$
\left\{1, e^{i\left(\omega_{1}+\omega_{2}\right)}, e^{i \omega_{2}}, e^{-i \omega_{1}}, e^{-i\left(\omega_{1}+\omega_{2}\right)}, e^{-i \omega_{2}}, e^{i \omega_{1}}\right\}
$$

has 6-fold axial symmetry, and hence, this 1-tap filter bank should be used as the initial symmetric filter bank. Let $D(\boldsymbol{\omega})$ be the diagonal matrix defined by (22). Then one can verify that $D(\boldsymbol{\omega})$ satisfies (39). Therefore, if $\left\{p, q^{(1)}, \cdots, q^{(6)}\right\}$ is given by

$$
\begin{aligned}
& {\left[p(\boldsymbol{\omega}), q^{(1)}(\boldsymbol{\omega}), \cdots, q^{(6)}(\boldsymbol{\omega})\right]^{T}=} \\
& \frac{1}{\sqrt{7}} C_{n} D(\boldsymbol{\omega}) C_{n-1} D(\boldsymbol{\omega}) \cdots C_{1} D(\boldsymbol{\omega}) C_{0} I_{0}(\boldsymbol{\omega}),
\end{aligned}
$$

where $n \in \mathbf{Z}_{+}$, and each $C_{k}, 0 \leq k \leq n$, satisfy (39), or equivalently, it has the form of

$$
C=\left[\begin{array}{lllllll}
c_{11} & c_{12} & c_{12} & c_{12} & c_{12} & c_{12} & c_{12} \\
c_{21} & c_{22} & c_{23} & c_{24} & c_{25} & c_{24} & c_{23} \\
c_{21} & c_{23} & c_{22} & c_{23} & c_{24} & c_{25} & c_{24} \\
c_{21} & c_{24} & c_{23} & c_{22} & c_{23} & c_{24} & c_{25} \\
c_{21} & c_{25} & c_{24} & c_{23} & c_{22} & c_{23} & c_{24} \\
c_{21} & c_{24} & c_{25} & c_{24} & c_{23} & c_{22} & c_{23} \\
c_{21} & c_{23} & c_{24} & c_{25} & c_{24} & c_{23} & c_{22}
\end{array}\right]
$$

then $\left\{p, q^{(1)}, \cdots, q^{(6)}\right\}$ satisfies (37). Therefore, the corresponding hexagonal filter bank has 6-fold axial symmetry.

The problem is that the structure in (40) hardly yields orthogonal or biorthogonal filter banks. To obtain bi(orthogonal) filter banks, we should factorize $\left[p(\boldsymbol{\omega}), q^{(1)}(\boldsymbol{\omega}), \cdots, q^{(6)}(\boldsymbol{\omega})\right]^{T}$ as $C\left(M^{T} \boldsymbol{\omega}\right)\left[p_{s}(\boldsymbol{\omega}), q_{s}^{(1)}(\boldsymbol{\omega}), \cdots, q_{s}^{(6)}(\boldsymbol{\omega})\right]^{T}$. However, if the basic block matrix is $C\left(M^{T} \boldsymbol{\omega}\right)$ for some $7 \times 7$ trigonometric polynomial matrix $C(\boldsymbol{\omega})$, then it will be hard to find simple $C(\boldsymbol{\omega})$ such that $C\left(M^{T} \boldsymbol{\omega}\right)$ satisfies (39). This is due to the fact that there is $19.1^{\circ}$ rotation of the axes of sublattice $\mathcal{G}_{7}$ with respect to the axes of $\mathcal{G}$. Because of these difficulties to construct a block structure of filter banks with 6-fold axial symmetry, in the following we consider another type of symmetry.

Definition 3: A hexagonal filter bank $\left\{P, Q^{(1)}, \cdots, Q^{(6)}\right\}$ is said to have pseudo 6-fold axial symmetry if the polyphase matrix $V(\boldsymbol{\omega})$ of its corresponding square filter bank $\left\{p, q^{(1)}, \cdots, q^{(6)}\right\}$ satisfies (39).

The left part of Fig. 12 shows a 49-tap lowpass filter with pseudo 6-fold axial symmetry, while the right part of Fig. 12 shows a 37-tap lowpass filter with 6-fold axial symmetry. Notice that the symmetry of the 49-tap lowpass filter is not only closely related to the symmetry structure of hexagonal lattice $\mathcal{G}$, but also related to the structure of sublattice $\mathcal{G}_{7}$. If the polyphase matrix $V(\boldsymbol{\omega})$ of $\left\{p, q^{(1)}, \cdots, q^{(6)}\right\}$ satisfies the first equation in (39), namely, $V\left(R_{1}^{-T} \boldsymbol{\omega}\right)=M_{0} V(\boldsymbol{\omega}) M_{0}^{-1}$, then by the facts $R_{1}^{T} M^{T} R_{1}^{-T}=M^{T}$ and $I_{0}\left(R_{1}^{-T} \boldsymbol{\omega}\right)=M_{0} I_{0}(\boldsymbol{\omega})$, we have

$$
\begin{aligned}
& {\left[p, q^{(1)}, \cdots, q^{(6)}\right]\left(R_{1}^{-T} \boldsymbol{\omega}\right)=\frac{1}{\sqrt{7}} V\left(M^{T} R_{1}^{-T} \boldsymbol{\omega}\right) I_{0}\left(R_{1}^{-T} \boldsymbol{\omega}\right)} \\
& =\frac{1}{\sqrt{7}}\left(M_{0} V\left(R_{1}^{T} M^{T} R_{1}^{-T} \boldsymbol{\omega}\right) M_{0}^{-1}\right) M_{0} I_{0}(\boldsymbol{\omega}) \\
& =\frac{1}{\sqrt{7}} M_{0} V\left(M^{T} \boldsymbol{\omega}\right) I_{0}(\boldsymbol{\omega})=M_{0}\left[p, q^{(1)}, \cdots, q^{(6)}\right](\boldsymbol{\omega}) .
\end{aligned}
$$

This, together with Proposition 2, implies that $\left\{p, q^{(1)}, \cdots, q^{(6)}\right\}$ has 6-fold rotational symmetry. Therefore, we also know that if a hexagonal filter bank has pseudo 6 -fold axial symmetry, then it has 6-fold rotational symmetry.

If the polyphase matrix $V(\boldsymbol{\omega})$ of $\left\{p, q^{(1)}, \cdots, q^{(6)}\right\}$ is given by the product of $C_{k} D(\boldsymbol{\omega})$ for some $C_{k}$ of the form (41), then $V(\boldsymbol{\omega})=V_{n}(\boldsymbol{\omega}) \cdots V_{1}(\boldsymbol{\omega}) V_{0}$ satisfies (39). Hence the 


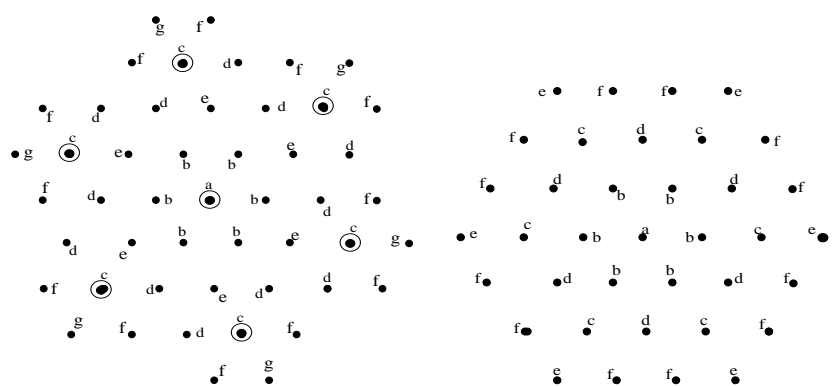

Fig. 12. Impulse response of lowpass filter with pseudo 6-fold axial symmetry (left) and impulse response of lowpass filter with 6-fold axial symmetry (right)

corresponding hexagonal filter bank $\left\{P, Q^{(1)}, \cdots, Q^{(6)}\right\}$ has pseudo 6-fold axial symmetry. In addition, if $C_{k}, 0 \leq k \leq n$ are orthogonal, then $\left\{P, Q^{(1)}, \cdots, Q^{(6)}\right\}$ is orthogonal. To summarize, we have the following result.

Theorem 4: If $\left\{p, q^{(1)}, \cdots, q^{(6)}\right\}$ is given by

$$
\begin{aligned}
& {\left[p(\boldsymbol{\omega}), q^{(1)}(\boldsymbol{\omega}), \cdots, q^{(6)}(\boldsymbol{\omega})\right]^{T}=} \\
& \quad \frac{1}{\sqrt{7}} C_{n} D\left(M^{T} \boldsymbol{\omega}\right) \cdots C_{1} D\left(M^{T} \boldsymbol{\omega}\right) C_{0} I_{0}(\boldsymbol{\omega}),
\end{aligned}
$$

where $n \in \mathbf{Z}_{+}, I_{0}(\boldsymbol{\omega})$ is defined by (21), and $C_{k}, 0 \leq$ $k \leq n$ are constant matrices of the form (41), then the FIR hexagonal filter bank $\left\{P, Q^{(1)}, \cdots, Q^{(6)}\right\}$ corresponding to $\left\{p, q^{(1)}, \cdots, q^{(6)}\right\}$ has pseudo 6 -fold axial symmetry. In addition, if $C_{k}, 0 \leq k \leq n$ are orthogonal, then $\left\{P, Q^{(1)}, \cdots, Q^{(6)}\right\}$ is an orthogonal filter bank.

For a matrix $C$ of the form (41), if it is orthogonal, then one can show directly or from (28) for the expression of orthogonal $B$ of the form (23) that the entries $c_{i j}$ of $C$ can be written as

$$
\begin{aligned}
& c_{11}=s_{0} \cos \xi, c_{12}=\frac{\sqrt{6}}{6} \sin \xi, c_{21}=s_{0} \frac{\sqrt{6}}{6} \sin \xi, \\
& c_{22}=\frac{1}{6}\left(s_{1}-\cos \xi+2 s_{2}+2 s_{3}\right), \\
& c_{23}=\frac{1}{6}\left(-s_{1}-\cos \xi+s_{2}-s_{3}\right), \\
& c_{24}=\frac{1}{6}\left(s_{1}-\cos \xi-s_{2}-s_{3}\right), \\
& c_{25}=\frac{1}{6}\left(-s_{1}-\cos \xi-2 s_{2}+2 s_{3}\right),
\end{aligned}
$$

where $s_{j}= \pm 1, j=0,1,2,3$, and $\xi \in \mathbb{R}$.

The block structure in (42) also yields biorthogonal FIR filter banks with pseudo 6-fold axial symmetry as shown in the next theorem.

Theorem 5: If $\left\{p, q^{(1)}, \cdots, q^{(6)}\right\}$ and $\left\{\widetilde{p}, \widetilde{q}^{(1)}, \cdots, \widetilde{q}^{(6)}\right\}$ are given by

$$
\begin{aligned}
& {\left[p(\boldsymbol{\omega}), q^{(1)}(\boldsymbol{\omega}), \cdots, q^{(6)}(\boldsymbol{\omega})\right]^{T}=} \\
& \quad \frac{1}{\sqrt{7}} C_{n} D\left(M^{T} \boldsymbol{\omega}\right) \cdots C_{1} D\left(M^{T} \boldsymbol{\omega}\right) C_{0} I_{0}(\boldsymbol{\omega}), \\
& {\left[\widetilde{p}(\boldsymbol{\omega}), \widetilde{q}^{(1)}(\boldsymbol{\omega}), \cdots, \widetilde{q}^{(6)}(\boldsymbol{\omega})\right]^{T}=} \\
& \quad \frac{1}{\sqrt{7}} C_{n}^{-T} D\left(M^{T} \boldsymbol{\omega}\right) \cdots C_{1}^{-T} D\left(M^{T} \boldsymbol{\omega}\right) C_{0}^{-T} I_{0}(\boldsymbol{\omega}),
\end{aligned}
$$

where $n \in \mathbf{Z}_{+}, I_{0}(\boldsymbol{\omega})$ is defined by (21), and $C_{k}, 0 \leq$ $k \leq n$ are nonsingular constant matrices of the form (41), then the FIR hexagonal filter banks $\left\{P, Q^{(1)}, \cdots, Q^{(6)}\right\}$ and $\left\{\widetilde{P}, \widetilde{Q}^{(1)}, \cdots, \widetilde{Q}^{(6)}\right\}$ corresponding to $\left\{p, q^{(1)}, \cdots, q^{(6)}\right\}$ and $\left\{\widetilde{p}, \widetilde{q}^{(1)}, \cdots, \widetilde{q}^{(6)}\right\}$ resp. are biorthogonal to each other and both of them have pseudo 6 -fold axial symmetry.

Example 5: Let $\left\{p, q^{(1)}, \cdots, q^{(6)}\right\}$ be the orthogonal filter banks with pseudo 6-fold axial symmetry given by (42) with $n=1: C_{1} D\left(M^{T} \boldsymbol{\omega}\right) C_{0} I_{0}(\boldsymbol{\omega})$, where $C_{0}, C_{1}$ are orthogonal matrices of the form (41) with their entries given by (43) for some $\xi_{0}, \xi_{1} \in \mathbb{R}$. With $s_{j}=1,0 \leq j \leq 3$ for $C_{0}, C_{1}$, by selecting $\xi_{0}=.9197818411, \xi_{1}=-.2634177990$, we have the (numerically) most Sobolev smooth scaling function $\phi$ which is in $W^{0.6523}$. In this case, the associated lowpass filter $p(\boldsymbol{\omega})$ has sum rule order 1 . The contours of the pictures of $\phi$ and $\psi^{(1)}$ are shown in Fig. 13 which may given us some idea about the pseudo 6-fold axial symmetry.
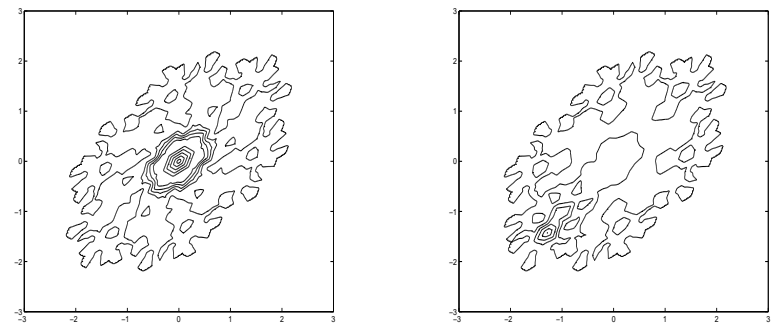

Fig. 13. Contours of $\phi$ (left) and $\psi^{(1)}$ (right)

Compared with filter banks with 6-fold rotational symmetry, filter banks with pseudo 6-fold axial symmetry have higher symmetry, and thus, they have fewer free parameters. Therefore, the above block structure of orthogonal/biorthogonal filter banks with pseudo 6-fold axial symmetry leads to scaling functions with less smoothness. Because of this, in this paper we would not provide more examples on the design of such filter banks based on the smoothness of scaling functions.

\section{APPENDIX A}

Selected parameters in Example 3: the selected $b_{i j}$ for $B_{0}$ are

$$
\begin{aligned}
& b_{11}=.6612620279, b_{12}=.4417207440, \\
& b_{21}=.2268020352, b_{22}=.2993798006, \\
& b_{23}=.1705704738, b_{24}=-.3949835111, \\
& b_{25}=-1.0052173436, b_{26}=.2334910801, \\
& b_{27}=.1629341515 ;
\end{aligned}
$$

and the selected $b_{i j}$ for $B_{1}$ are

$$
\begin{aligned}
& b_{11}=.7505582508, b_{12}=-.0869709994 \\
& b_{21}=-.0675087754, b_{22}=.1148885367, \\
& b_{23}=.0883847501, b_{24}=-.2784941222, \\
& b_{25}=-.8412833742, b_{26}=.2934347543, \\
& b_{27}=-.1050874592 .
\end{aligned}
$$

\section{APPENDIX B}

Selected parameters for Bio_2 in Example 4: the selected $b_{i j}$ for $B_{0}$ are

$$
\begin{aligned}
& b_{11}=.2431365209, b_{12}=.5340175677, \\
& b_{21}=.8329155977, b_{22}=.2905157027, \\
& b_{23}=.0087935205, b_{24}=-.7634995587, \\
& b_{25}=-.9384099041, b_{26}=-.2165868980, \\
& b_{27}=.2722450171 ;
\end{aligned}
$$


the selected $b_{i j}$ for $B_{1}$ are

$$
\begin{aligned}
& b_{11}=.5365830002, b_{12}=-.1191592023, \\
& b_{21}=-.3360950603, b_{22}=.0771447245, \\
& b_{23}=-.1385489895, b_{24}=-.5902499286, \\
& b_{25}=-.8324734053, b_{26}=-.6045485018, \\
& b_{27}=-.1527809072 ;
\end{aligned}
$$

and the selected $b_{i j}$ for $B_{2}$ are

$$
\begin{aligned}
& b_{11}=1.1918768947, b_{12}=-.0797323587, \\
& b_{21}=.0102962235, b_{22}=1.2596364364, \\
& b_{23}=.5358680341, b_{24}=.7336434639, \\
& b_{25}=.2152676640, b_{26}=.0555687397, \\
& b_{27}=.7489785014 .
\end{aligned}
$$

Acknowledgments. The author thanks Dale K. Pounds for his kind help to create Figs 9-11. The author also thanks the anonymous reviewers for making valuable suggestions that significantly improved the presentation of the paper.

\section{REFERENCES}

[1] D.P. Petersen and D. Middleton, "Sampling and reconstruction of wave-number-limited functions in N-dimensional Euclidean spaces", Information and Control, vol. 5, no. 4, pp. 279-323, Dec. 1962.

[2] R.M. Mersereau, "The processing of hexagonal sampled twodimensional signals", Proc. IEEE, vol. 67, no. 6, pp. 930-949, Jun 1979.

[3] R.C. Staunton and N. Storey, "A comparison between square and hexagonal sampling methods for pipeline image processing", In Proc. of SPIE Vol. 1194, Optics, Illumination, and Image Sensing for Machine Vision IV, 1990, pp. 142-151.

[4] G. Tirunelveli, R. Gordon, and S. Pistorius, "Comparison of square-pixel and hexagonal-pixel resolution in image processing", in Proceedings of the 2002 IEEE Canadian Conference on Electrical and Computer Engineering, vol. 2, May 2002, pp. 867-872.

[5] D. Van De Ville, T. Blu, M. Unser, W. Philips, I. Lemahieu, and R. Van de Walle, "Hex-splines: a novel spline family for hexagonal lattices", IEEE Tran. Image Proc., vol. 13, no. 6, pp. 758-772, Jun. 2004.

[6] L. Middleton and J. Sivaswarmy, Hexagonal Image Processing: A Practical Approach, Springer, 2005.

[7] X.J. He and W.J. Jia, "Hexagonal structure for intelligent vision", in Proceedings of the 2005 First International Conference on Information and Communication Technologies, Aug. 2005, pp. 52-64.

[8] X.Q. Zheng, "Efficient Fourier Transforms on Hexagonal Arrays", Ph.D Dissertation, University of Florida, 2007.

[9] R.C. Staunton, "The design of hexagonal sampling structures for image digitization and their use with local operators", Image and Vision Computing, vol. 7, no. 3, pp. 162-166, Aug. 1989.

[10] L. Middleton and J. Sivaswarmy, "Edge detection in a hexagonal-image processing framework", Image and Vision Computing, vol. 19, no. 14, pp. 1071-1081, Dec. 2001.

[11] A.F. Laine, S. Schuler, J. Fan, and W. Huda, "Mammographic feature enhancement by multiscale analysis", IEEE Trans. Med. Imaging, vol. 13, no. 4, pp. 725-740, Dec. 1994.

[12] A.F. Laine and S. Schuler, "Hexagonal wavelet representations for recognizing complex annotations", in Proceedings of the IEEE Conference on Computer Vision and Pattern Recognition, Seattle, WA, Jun. 1994 pp. 740-745.

[13] A.P. Fitz and R. Green, "Fingerprint classification using hexagonal fast Fourier transform", Pattern Recognition, vol. 29, no. 10, pp. 1587-1597, 1996.

[14] S. Periaswamy, "Detection of microcalcification in mammograms using hexagonal wavelets", M.S. thesis, Dept. of Computer Science, Univ. of South Carolina, Columbia, SC, 1996

[15] R.C. Staunton, "One-pass parallel hexagonal thinning algorithm", IEE Proceedings on Vision, Image and Signal Processing, vol. 148, no. 1, pp. 45-53, Feb. 2001
[16] A. Camps, J. Bara, I.C. Sanahuja, and F. Torres, "The processing of hexagonally sampled signals with standard rectangular techniques: application to 2-D large aperture synthesis interferometric radiometers", IEEE Trans. Geoscience and Remote Sensing, vol. 35, no. 1, pp. 183190, Jan. 1997.

[17] E. Anterrieu, P. Waldteufel, and A. Lannes, "Apodization functions for 2-D hexagonally sampled synthetic aperture imaging radiometers", IEEE Trans. Geoscience and Remote Sensing, vol. 40, no. 12, pp. 2531-2542, Dec. 2002.

[18] D. White, A.J. Kimberling, and W.S. Overton, "Cartographic and geometric components of a global sampling design for environmental monitoring", Cartography and Geographic Information Systems, vol. 19, no. 1, pp. 5-22, 1992

[19] K. Sahr, D. White, and A.J. Kimerling, "Geodesic discrete global grid systems", Cartography and Geographic Information Science, vol. 30, no. 2, pp. 121-134, Apr. 2003.

[20] M.J.E. Golay, "Hexagonal parallel pattern transformations", IEEE Trans. Computers, vol. 18, no. 8, pp.733-740, Aug. 1969.

[21] P.J. Burt, "Tree and pyramid structures for coding hexagonally sampled binary images", Computer Graphics and Image Proc., vol. 14, no. 3 , pp. $271-80,1980$

[22] L. Kobbelt, “ $\sqrt{3}$-subdivision", in SIGGRAPH Computer Graphics Proceedings, pp. 103-112, 2000.

[23] U. Labsik and G. Greiner, "Interpolatory $\sqrt{3}$-subdivision", Computer Graphics Forum, vol. 19, no. 3, pp. 131-138, Sep. 2000.

[24] Q.T. Jiang and P. Oswald, "Triangular $\sqrt{3}$-subdivision schemes: the regular case", J. Comput. Appl. Math., vol. 156, no. 1, pp. 47-75, Jul. 2003.

[25] Q.T. Jiang, P. Oswald, and S.D. Riemenschneider, " $\sqrt{3}$-subdivision schemes: maximal sum rules orders", Constr. Approx., vol. 19, no. 3, pp. 437-463, 2003.

[26] P. Oswald and P. Schröder, "Composite primal/dual $\sqrt{3}$-subdivision schemes", Comput. Aided Geom. Design, vol. 20, no. 3, pp. 135-164, Jun. 2003.

[27] C.K. Chui and Q.T. Jiang, "Matrix-valued symmetric templates for interpolatory surface subdivisions, I: regular vertices", Appl. Comput. Harmonic Anal., vol. 19, no. 3, pp. 303-339, Nov. 2005.

[28] P. Oswald, "Designing composite triangular subdivision schemes", Comput. Aided Geom. Design, vol. 22, no. 7, pp. 659-679, Oct. 2005.

[29] C.K. Chui, Q.T. Jiang, and R.N. Ndao, "Triangular $\sqrt{7}$ and quadrilateral $\sqrt{5}$ subdivision schemes: regular case", J. Math. Anal. Appl., vol. 338, no. 2, pp. 1204-1223, Feb. 2008

[30] E. Simoncelli and E. Adelson, "Non-separable extensions of quadrature mirror filters to multiple dimensions", Proceedings of the IEEE, vol. 78 , no. 4, pp. 652-664, Apr. 1990.

[31] E.A. Adelson, E. Simoncelli, and R. Hingorani, "Orthogonal pyramid transforms for image coding", In SPIE Visual Communications and Image Processing II (1987), vol. 845, 1987, pp. 50-58.

[32] H. Xu, W.-S. Lu, and A. Antoniou, "A new design of 2-D non-separable hexagonal quadrature-mirror-filter banks", in Proc. CCECE, Vancouver, Sep. 1993, pp. 35-38

[33] A. Cohen and J.-M. Schlenker, "Compactly supported bidimensional wavelets bases with hexagonal symmetry", Constr. Approx., vol. 9, no. 2/3, pp. 209-236, Jun. 1993.

[34] J.D. Allen, "Coding transforms for the hexagon grid", Ricoh Calif. Research Ctr., Technical Report CRC-TR-9851, Aug. 1998.

[35] J.D. Allen, "Perfect reconstruction filter banks for the hexagonal grid", In Fifth International Conference on Information, Communications and Signal Processing 2005, Dec. 2005, pp. 73-76.

[36] Q.T. Jiang, "FIR filter banks for hexagonal data processing", IEEE Trans. Image Proc., in press.

[37] L. Middleton and J. Sivaswarmy, "Framework for practical hexagonalimage processing", J. Electronic Imaging, vol. 11, no. 1, pp. 104-114, Jan. 2002.

[38] A.B. Watson and A. Ahumada, Jr., "A hexagonal orthogonal-oriented pyramid as a model of image presentation in visual cortex", IEEE Trans. Biomed. Eng., vol. 36, no. 1, pp. 97-106, Jan. 1989.

[39] A. Lundmark, N. Wadströmer, and H. Li, "Hierarchical subsample giving fractal region”, IEEE Trans. Image Proc., vol. 10, no. 1, pp. 167-173, Jan. 2001.

[40] S. Schuler and A.F. Laine, "Hexagonal QMF banks and wavelets", a chapter in Time Frequency and Wavelets in Biomedical Signal Processing, M. Akay (Ed.), IEEE Press, 1997.

[41] C. de Boor, K. Höllig, and S. Riemenschneider, Box splines, SpringerVerlag, New York, 1993 
[42] C.K. Chui and Q.T. Jiang, "Multivariate balanced vector-valued refinable functions", in Mordern Development in Multivariate Approximation, ISNM 145, Birhhäuser Verlag, Basel, 2003, pp. 71-102.

[43] A. Cohen and I. Daubechies, "A stability criterion for biorthogonal wavelet bases and their related subband coding scheme", Duke Math. J., vol. 68, no. 2, pp. 313-335, 1992.

[44] R.Q. Jia, "Convergence of vector subdivision schemes and construction of biorthogonal multiple wavelets", In Advances in Wavelets, SpringerVerlag, Singapore, 1999, pp. 199-227.

[45] Q.T. Jiang, "Orthogonal multiwavelets with optimum time-frequency resolution”, IEEE Trans. Signal Proc., vol. 46, no. 4, pp. 830-844, Apr. 1998.

[46] Q.T. Jiang, "On the design of multifilter banks and orthonormal multiwavelet bases", IEEE Trans. Signal Proc., vol. 46, no. 12, pp. 3292 3303, Dec. 1998.

[47] T. Xia and Q.T. Jiang, "Optimal multifilter banks: design, related symmetric extension transform and application to image compression", IEEE Trans. Signal Proc., vol. 47, no. 7, 1878-1889, Jul. 1999.

[48] R.Q. Jia, "Approximation properties of multivariate wavelets", Math. Comp., vol. 67, no. 222, pp. 647-665, Apr. 1998.

[49] R.Q. Jia and S.R. Zhang, "Spectral properties of the transition operator associated to a multivariate refinement equation", Linear Algebra Appl., vol. 292, no. 1, pp. 155-178, May 1999.

[50] R.Q. Jia and Q.T. Jiang, "Spectral analysis of transition operators and its applications to smoothness analysis of wavelets", SIAM J. Matrix Anal. Appl., vol. 24, no. 4, pp. 1071-1109, 2003.

[51] J. Kovačević and M. Vetterli, "Nonspeparable multidimensional perfect reconstruction filter banks and wavelet bases for $\mathbb{R}^{n}$ ", IEEE Trans. Inform. Theory, vol. 38, no. 2, pp. 533-555.

[52] K. Gröchenig and W.R. Madych, "Multiresolution analysis, Haar bases, and self-similar tilings of $\mathbb{R}^{n}$,, IEEE Trans. Inform. Theory, vol. 38, no. 2, pp. 556-568.

\begin{tabular}{|c|} 
\\
\\
PLACE \\
PHOTO \\
HERE \\
\end{tabular}

Qingtang Jiang received the B.S. and M.S. degrees from Hangzhou University, Hangzhou, China, in 1986 and 1989, respectively, and the Ph.D. degree from Peking University, Beijing, China, in 1992, all in mathematics. He was with Peking University from 1992 to 1995 . He was an NSTB postdoctoral fellow and then a research fellow at the National University of Singapore from 1995 to 1999. Before he joined the University of Missouri-St. Louis, in 2002, he held visiting positions at University of Alberta, Canada, and West Virginia University, Morgantown. $\mathrm{He}$ is now a Professor in the Department of Math and Computer Science, University of Missouri-St. Louis. His current research interests include timefrequency analysis, wavelet theory and its applications, filter bank design, signal classification, image processing, and surface subdivision. 